$\underline{\text { Preprint typeset in JHEP style - HYPER VERSION }}$

La Plata Th-09/01

February, 2010

\title{
Gauge invariant perturbation theory and non-critical string models of Yang-Mills theories
}

\author{
Adrián R. Lugo, Mauricio B. Sturla \\ Instituto de Física de La Plata (IFLP) - Departamento de Física \\ Facultad de Ciencias Exactas, Universidad Nacional de La Plata \\ C. C. 67, (1900) La Plata, Argentina. \\ E-mail: lugo@fisica.unlp.edu.ar, sturla@fisica.unlp.edu.ar
}

\begin{abstract}
We carry out a gauge invariant analysis of certain perturbations of $D-2$ branes solutions of low energy string theories. We get generically a system of second order coupled differential equations, and show that only in very particular cases it is possible to reduce it to just one differential equation. Later, we apply it to a multi-parameter, generically singular family of constant dilaton solutions of non-critical string theories in $D$ dimensions, a generalization of that recently found in arXiv:0709.0471 [hep-th]. According to arguments coming from the holographic gauge theory-gravity correspondence, and at least in some region of the parameters space, we obtain glue-ball spectra of Yang-Mills theories in diverse dimensions, putting special emphasis in the scalar metric perturbations not considered previously in the literature in the non critical setup. We compare our numerical results to those studied previously and to lattice results, finding qualitative and in some cases, tuning properly the parameters, quantitative agreement. These results seem to show some kind of universality of the models, as well as an irrelevance of the singular character of the solutions. We also develop the analysis for the T-dual, non trivial dilaton family of solutions, showing perfect agreement between them.
\end{abstract}

KEYWORDs: Non-critical supergravity, AdS/CFT correspondence. 


\section{Contents}

1. Introduction 2

2. The family of solutions 3

3. Wilson loops and confinement 5

4. The gauge-invariant perturbative setup 8

4.1 The equation for dilatonic fluctuations 11

4.2 The equation for RR one-form fluctuations $\overline{12}$

4.3 The equation for the metric fluctuations 13

5. Holographic models of $d$ dimensional Yang-Mills theories 14

5.1 Dilatonic fluctuations 14

5.2 RR gauge field: transverse fluctuations 14

5.3 RR gauge field: longitudinal fluctuations 14

5.4 Metric: transverse fluctuations

5.5 Metric: longitudinal fluctuations 15

5.6 Metric: scalar fluctuations 15

6. Glue-ball spectra of $3 D$ Yang-Mills theories. 18

6.1 Spectrum from dilatonic fluctuations.

6.2 Spectra from RR 1-form fluctuations. 21

6.3 Glueball spectra from metric perturbations 22

7. Glueball spectra of $4 D$ Yang-Mills theories. 23

7.1 Spectrum from dilatonic fluctuations. 25

7.2 Spectra from RR 1-form fluctuations. 25

7.3 Glueball spectra from metric perturbations 26

8. Summary of results and discussion 28

A. Some useful formulae. 33

A.1 Computation of the tensor $A_{A B}$

A.2 A short derivation of the solutions. 35

B. The fluctuations in the T-dual solutions. 36

B.1 The family of solutions. 36

B.2 The equations for the fluctuations in e-frame 37

B.3 Holographic models of $d$ dimensional Yang-Mills theories 40

B.4 Metric: transverse fluctuations 40

B.5 Metric: longitudinal fluctuations 40 


\section{Introduction}

Since the equivalence between type IIB superstring theory on $A d S_{1,4} \times S^{5}$ and $\mathcal{N}=4$ superconformal Yang-Mills theory in four dimensions was put on firm grounds [1] [2] [3], many works has been devoted to possible extensions of this gauge/gravity correspondence to general conformal setups, and non conformal (even confining) and less supersymmetric ones [4] [5]. In particular, soon after Maldacena' s work, Witten proposed a possible path to study non-supersymmetric, pure Yang-Mills (Y-M) theories at large number of colors $N$, starting from finite temperature field theories and their conjectured gravity duals, i.e. AdS black holes like solutions [6]. Among other things, he showed in a general context properties like confinement and the existence of a mass gap, and how to compute the spectrum of bound states of gluons ("glue-balls") (see also [7]). Since then, many papers were devoted to the calculation of these spectra, mainly in three and four dimensions [8] [9] [10] [1] [12] 13. [14.

In most of these cases two main related problems are present. On one hand, the validity of supergravity computations breaks down when we attempt to reach the field theory limit, and so we must content ourselves with calculations at finite cut-off. On the other hand, the spectra of glue-balls, whose masses should be of the order of the typical scale of the theory $\left(\Lambda_{Q C D}\right)$, result "contaminated" by the presence of Kaluza-Klein modes with masses of the same order coming from the extra dimensions of 10-dimensional superstring theories, that certainly are not present in pure Y-M. An attempt to overcome these problems (or part of them) could be to study holographic duals in the context of string theories in dimensions less that ten [15]. More that a decade ago, Kutasov and Seiberg constructed tachyonfree superstring theories in even dimensions $D<10$, the so-called type II non-critical superstrings (NCS) [16] [17]. Recently, in reference [18] Kuperstein and Sonnenschein (K-S) investigated the supergravity equations of motion associated with these non-critical string theories that incorporate RR forms, and derived several classes of solutions. In particular, they found analytic backgrounds with a structure of $A d S_{1, p+1} \times S^{k}$, and numerical solutions that asymptote a linear dilaton with a topology of $\Re^{1, D-3} \times \Re \times S^{1}$. Unfortunately, for all these solutions the curvature in string units is proportional to $c=10-D$ and it cannot be reduced by taking a large $N$ limit like in the critical case. This means that the supergravity approximation can not be fully justifiable. They conjectured however, that higher order corrections would modify the radii, while leaving the geometrical structure of the background unchanged. In the light of the by now well-established gauge-gravity correspondence, they took one step forward and used the near extremal $A d S_{1,5}$ background to extract dynamical properties of four dimensional confining theories [19]. This $A d S_{1,5}$ model, which is a member of the family of solutions mentioned above, includes a zero form field strength that may be associated with a $D 4$ brane in a similar way to the $D 8$ brane 
of the critical type IIA superstring theory. The counterpart on the gauge theory side of the introduction of near extremality, i.e. the incorporation of a black hole, is given by the compactification of the euclidean time coordinate on a circle and the imposition of anti-periodic boundary conditions on the fermions, that is, to consider the five dimensional theory living on the brane at finite temperature [6].

Later on, it was discovered in reference [21] a whole family of AdS black hole-like solutions in arbitrary dimension $D$ that includes the K-S solution previously mentioned. These families present a constant dilaton, includes a $D \equiv p+2$ form field strength proportional to an integer $N$ associated with the presence of $N D p$ branes, and are parameterized by certain exponents that satisfy a set of constraints. Although these solutions are Einstein spaces, they present a singularity. It was conjectured in 21] to be T-dual to solutions of $N$ black $D(p-1)$ branes placed in the $\mathcal{N}=2$ superconformal linear dilaton vacuum $\Re^{1, p-1} \times \Re \times S^{1}$ (a fact proved by numerical analysis in 18 in the case of the T-dual solution to the $A d S_{1, D-1}$ background).

In the spirit of [6], and along the lines of [19], we consider in this paper holographic models of $d=3,4$ dimensional gauge theories based on these families of solutions. After analyzing the issue of confinement, we devote our efforts to the extraction of the glueball spectra associated with the fluctuations of the dilaton, the graviton and the R-R one form. For carrying out this task, and at difference of computations usually found in the literature, we prefer to develop a gauge-invariant framework to analyze numerically the resulting, generically coupled, second order systems of differential equations. We put special emphasis in the dependence of the results on the set of exponents that label the families.

The organization of the paper is as follows. In Section 2 we present the non-critical families to be analyzed along the paper, and discuss the salient features of them. In Section 3 we briefly describe the Wilson loop computation, and discuss the issue of confinement for these models. Section 1 is devoted to present the gauge-invariant perturbative formalism. In Section 5 we discuss the models to be considered. In Section 6 we present the results of the calculation of the glue-ball spectra in three-dimensional gauge theory in the context of non-critical supergravity, while that in Section 0 we carry out a similar analysis for models of four-dimensional gauge theory. In Section 8 we present a discussion of our results. We make an analysis of the spectra obtained and compare them with the lattice $Y M_{3}$ and $Y M_{4}$ calculations. We find that there is qualitative and quantitative agreement between both results. Two appendices are added. In Appendix A we collect relevant formulae as well as a derivation of the family of constant dilaton solutions considered in the paper. In Appendix B we present an analysis of the perturbations for the T-dual, non trivial dilaton family, showing perfect agreement with the spectra obtained in the constant dilaton family case analyzed in the main of the paper.

\section{The family of solutions}

Let us consider the bosonic part of the low energy effective action of non critical (super) 
strings in $D$ dimensions, that in string frame reads,

$$
S\left[G, \Phi, A_{q+1}\right]=\frac{1}{2 \kappa_{D}^{2}} \int \epsilon_{G}\left(e^{-2 \Phi}\left(R[G]+4(D \Phi)^{2}+\Lambda^{2}\right)-\frac{1}{2} \sum_{q} e^{2 b_{q} \Phi}\left(F_{q+2}\right)^{2}\right)
$$

where $F_{q+2}=d A_{q+1}$ is the field strength of the gauge field form $A_{q+1}, b_{q}=0(-1)$ for RR (NSNS) forms, and the possible $q$ 's depend on the theory. The volume element is $\epsilon_{G}=\omega^{0} \wedge \cdots \wedge \omega^{D-1}=d^{D} x \sqrt{-\operatorname{det} G}$, with $\left\{\omega^{A}\right\}$ the vielbein.

The cosmological constant $\Lambda^{2}(>0)$ is identified in (super) string theories with $\left(\frac{10-D}{\alpha^{\prime}}\right)$ $\frac{2(26-D)}{3 \alpha^{\prime}}$, where $T_{s}=\left(2 \pi \alpha^{\prime}\right)^{-1}$ is the string tension, while that the $D$-dimensional Newton constant $\kappa_{D}^{2} \sim \alpha^{\prime \frac{D}{2}-1}$.

It is possible to show that the equations of motion that follow from (2.1) are solved by,

$$
\begin{aligned}
l_{0}^{-2} G & =\sum_{a=0}^{D-2} u^{2} f(u)^{a_{a}} d x^{a 2}+\frac{d u^{2}}{u^{2} f(u)} \quad ; \quad f(u) \equiv 1-\left(\frac{u_{0}}{u}\right)^{D-1} \\
e^{\Phi} & =\frac{2}{\sqrt{D}} \frac{\Lambda}{\left|Q_{D-2}\right|} \\
F_{D} & =(-)^{D} Q_{D-2} \epsilon_{G} \Longleftrightarrow * F_{D}=(-)^{D-1} Q_{D-2}
\end{aligned}
$$

where $l_{0}=\sqrt{D(D-1)} \Lambda^{-1}, u_{0}$ is an arbitrary scale, and the following constraints on the exponents must hold,

$$
\sum_{a=0}^{D-2} a_{a}=1 \quad, \quad \sum_{a=0}^{D-2} a_{a}^{2}=1
$$

A short derivation is given in Appendix A.

This is a generalized version of the constant dilaton family obtained in 21] by performing a T-duality transformation of a non trivial dilaton family. In this paper we will focus on these solutions; an analysis of the non trivial dilaton ones is presented in Appendix B. The solutions can be interpreted as the near horizon limit of a D- $(D-2)$ black-brane extended along the $x$-coordinates. They are Einstein spaces with Ricci tensor,

$$
R_{A B}=-\frac{D-1}{l_{0}^{2}} G_{A B} \quad, \quad R=-\Lambda^{2}
$$

Furthermore, all of them are asymptotic at large $u \gg u_{0}$ to $A d S_{1, D-1}$ space. However, the only solution strictly regular also in the IR region $u \rightarrow u_{0}{ }^{+}$, corresponds to take one of the $a_{a}^{\prime} s$ equal to one, the other ones zero. Explicitly,

$$
l_{0}^{-2} G=u^{2}\left(\eta_{1, D-3}+f(u) d \tau^{2}\right)+\frac{d u^{2}}{u^{2} f(u)}
$$

This can be verified from the computation of the fourth order invariant,

$$
\begin{aligned}
& \left(\frac{l_{0}}{D-1}\right)^{4} \Re^{A B C D} \Re_{A B C D}=\frac{1}{8}\left(4\left(1-s_{3}\right)-1+s_{4}\right) f(u)^{-2} \\
+ & \frac{1}{2}\left(-\frac{2 D}{D-1}\left(1-s_{3}\right)+1-s_{4}\right) f(u)^{-1}
\end{aligned}
$$




$$
+\frac{9}{(D-1)^{2}}+\frac{1}{2}\left(1-\frac{12}{D-1}+\frac{6}{D-1}\left(1-s_{3}\right)-\frac{3}{2}\left(1-s_{4}\right)\right)+o(f(u))
$$

where $s_{n} \equiv \sum_{a} a_{a}{ }^{n}$. Clearly, the only way of cancelling the dangerous terms is to impose $s_{3}=s_{4}=1$; together with the constraints $s_{1}=s_{2}=1$ we get the solution (2.5) as the only possibility. It is the $A d S_{1, D-1}$ Schwarzchild black hole recently derived in [18, and used in [19] as a model (for $D=6$ ) of four dimensional Y-M theory. A further imposition to avoid a conical singularity in the IR is to impose the periodicity condition,

$$
\tau \sim \tau+\beta \quad, \quad \beta \equiv \frac{4 \pi}{D-1} \frac{1}{u_{0}}
$$

This periodicity is usual in solutions that we associate to field theories at finite temperature $\beta^{-1}[6]$.

What about the other solutions? As discussed in the introduction, the IR behavior of the curvature make the solutions not to be under control anyway, as it happens in critical string theories, For any member of the constant dilaton family yields an effective string coupling constant,

$$
g_{s}=e^{\Phi}=\sqrt{\frac{10}{D}-1} \frac{4 \pi}{\left|Q_{p}\right|} \sim \frac{1}{N}
$$

where $N$ is the number of $\mathrm{D}(D-2)$-branes; then it will be small, and so perturbative string theory will be valid for any solution of the family, provided that we take a large $N$ limit. Furthermore, following [18] the computation on the gravity side of the number of degrees of freedom ("entropy") in the UV region yields,

$$
S_{\text {gravity }} \sim \frac{N^{2}}{\delta^{D-2}}
$$

where $\delta$ is an IR cutoff; this is exactly the result we expect for a $D-1$ dimensional $U(N)$ gauge theory with UV cutoff $\delta^{-1}[26]$. So, we will consider the general case with arbitrary exponents (subject to the constraints (2.3)), and therefore we will have free parameters as well as additional Kaluza-Klein (KK) directions. Among the objectives of the paper is to studying the dependence of the spectra on the exponents.

\section{Wilson loops and confinement}

It is known since some time ago [27], [28] that the stringy description of a Wilson loop is in terms of a string whose end-points are attached at two points on the boundary of the AdS-like black hole space-time, that represent a quark anti-quark pair from the point of view of the gauge theory. Then, we will be interested in strings that end at $u=u_{\infty}(\rightarrow$ $\infty), x^{1}= \pm L / 2$, where $x^{1}$ denotes one of the $p$ spatial directions. In [29] the classical energy of the Wilson loop associated with a background metric of the form,

$$
G=G_{00} d x^{02}+\sum_{i, j=1}^{p} G_{i i} \delta_{i j} d x^{i} d x^{j}+C(u)^{2} d u^{2}+G^{\perp}
$$


with a general dependence on the radial direction was written down, where $G^{\perp}$ is orthogonal to the $\left(x^{0}, x^{i}, u\right)$-directions. Let us briefly sketch the computation.

Let $\left(\sigma^{\alpha}\right)=(t, \sigma)$ parameterize the string world-sheet. In the static gauge $X^{0}(t, \sigma)=$ $t \in \Re, X^{1}(t, \sigma)=\sigma \in\left[-\frac{L}{2}, \frac{L}{2}\right]$, let us consider the static configuration of a string defined by $u(t, \sigma)=u(\sigma)=u(-\sigma) \in\left[u_{0}, u_{\infty}\right]$, and the rest of the coordinates fixed. The NambuGoto lagrangian is,

$$
L[X]=T_{s} \int_{-\frac{L}{2}}^{\frac{L}{2}} d \sigma \sqrt{-\operatorname{det} h_{\alpha \beta}(\sigma)}=T_{s} \int_{-\frac{L}{2}}^{\frac{L}{2}} d \sigma \sqrt{F(u)^{2}+G(u)^{2} u^{\prime}(\sigma)^{2}}
$$

Here $h_{\alpha \beta}(\sigma) \equiv G_{M N}(X) \partial_{\alpha} X^{M}(\sigma) \partial_{\beta} X^{N}(\sigma)$ is the induced metric, and the functions $F$ and $G$ are defined by,

$$
F(u) \equiv\left|G_{00} G_{i i}\right|^{\frac{1}{2}} \quad, \quad G(u) \equiv\left|G_{00} C^{2}\right|^{\frac{1}{2}}
$$

For a minimal action configuration,

$$
\frac{F(u) \partial_{u} F(u)+G(u) \partial_{u} G(u) u^{\prime}(\sigma)}{\sqrt{F(u)^{2}+G(u)^{2} u^{\prime}(\sigma)^{2}}}=\left(\frac{G(u)^{2} u^{\prime}(\sigma)^{2}}{\sqrt{F(u)^{2}+G(u)^{2} u^{\prime}(\sigma)^{2}}}\right)^{\prime}
$$

The separation between quarks and energy that follow from (3.2), (3.4) are,

$$
\begin{aligned}
& L=2 \int_{u_{m}}^{u_{\infty}} d u \frac{G(u)}{F(u)}\left(\frac{F(u)^{2}}{F\left(u_{m}\right)^{2}}-1\right)^{-\frac{1}{2}} \\
& E=T_{s}\left(F\left(u_{m}\right) L+2 \int_{u_{m}}^{u_{\infty}} d u G(u) \sqrt{1-\frac{F\left(u_{m}\right)^{2}}{F(u)^{2}}}\right)
\end{aligned}
$$

where $u_{m}=u\left(\sigma_{m}\right) \geq u_{0}$ for (admitting it is unique) $\sigma_{m}$ such that $u^{\prime}\left(\sigma_{m}\right)=0\left(\sigma_{m}=\right.$ $0, u_{m}=u(0)$, for our configuration), and $F(u) \geq F\left(u_{m}\right)$. However the expression for the energy diverges for $u_{\infty} \rightarrow \infty$ due to the contribution of the self-energy (mass) of the two quarks [27], each one represented for long strings puncturing the boundary $u=u_{\infty}$ at $x^{1}= \pm \frac{L}{2}$ and extended along the $u$-direction,

$$
m_{q}=T_{s} \int_{u_{0}}^{u_{\infty}} d u G(u)
$$

By subtracting the masses we get the binding energy as,

$$
\begin{aligned}
& V(L) \equiv E-2 m_{q}=T_{s}\left(F\left(u_{m}\right) L-2 K(L)\right) \\
& K(L)=\int_{u_{m}}^{u_{\infty}} d u G(u)\left(1-\sqrt{1-\frac{F\left(u_{m}\right)^{2}}{F(u)^{2}}}\right)+\int_{u_{0}}^{u_{m}} d u G(u)
\end{aligned}
$$

From the analysis of (3.7) it follows that sufficient condition for an area law behavior is that $F(u)$ has a minimum at $u=u_{\min } \geq u_{0}$ and $F\left(u_{\min }\right)>0$, or that $G(u)$ diverges at 
some $u_{\text {div }} \geq u_{0}$ and $F\left(u_{\text {div }}\right)>0{ }^{1}$. In this case the quark anti-quark potential of the dual gauge theory results linear in the separation distance $L$; it follows from (3.7) that,

$$
\lim _{L \rightarrow \infty} \frac{V(L)}{L}=\sigma_{s} \quad, \quad \sigma_{s}=T_{s} F\left(u_{\min }\right) \quad \text { or } \quad T_{s} F\left(u_{\text {div }}\right)
$$

where $\sigma_{s}$ is the (YM) string tension. Specializing to our family of solutions, it is not difficult to see that $F(u)=\left.\left(l_{0} u_{0}\right)^{2} h(x)\right|_{x=\frac{u^{2}}{u_{0}{ }^{2}}}$ presents a minimum at $u=u_{\min }$ if

$$
\gamma \equiv-\frac{1}{2}(a+\tilde{a})>0 \Longrightarrow u_{\min }=u_{0}\left(1+\frac{D-1}{2} \gamma\right)^{\frac{1}{D-1}}
$$

The function $h(x)=x\left(1-x^{-\frac{D-1}{2}}\right)^{-\gamma}$ is displayed in Figure (1) for different values of $\gamma$ and $D$.

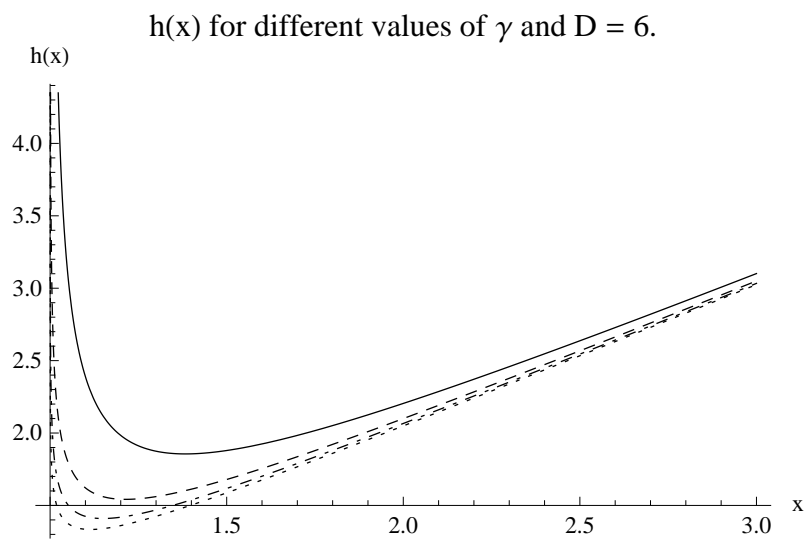

Figure 1: The plot shows $h(x)$ as a function of $x$, where $\gamma$ is between $1 / 8$ and $1 / 2$. The dotted line corresponds to $\gamma=1 / 8$, while the solid line corresponds to $\gamma=1 / 2$.

We conclude that if we restrict the space of parameters to the region $\gamma>0$, then the dual gauge theory should be (classically) confining, with a string tension given by,

$$
\sigma_{s} \equiv \tilde{\sigma}_{s} u_{0}^{2} \quad, \quad \tilde{\sigma}_{s}=\left(\frac{D-1}{2}\right)^{\frac{D+1}{D-1}} \frac{D}{\pi(10-D)} \frac{\left(\gamma+\frac{2}{D-1}\right)^{\gamma+\frac{2}{D-1}}}{\gamma^{\gamma}}
$$

\footnotetext{
${ }^{1}$ We refer the reader to 22] for the complete statement of the theorem, that includes other hypothesis about the behavior on $F$ and $G$ (that are fulfilled by our family of solutions). We remark that in (3.5) the separation $L$ should be considered as fixed, and $u_{m}$ though as a function of it. Moreover, $u_{\min }$ certainly does not depend on $u_{m}$, but $u_{m} \geq u_{\text {min }}$ must hold for the configuration to exist. It is not difficult to show for (3.3) that to taking $u_{m} \rightarrow u_{\text {min }}{ }^{+}$is equivalent to taking the limit $L \rightarrow \infty$, which is just that condition that leads to the definition of the string tension $\sigma_{s}$ in (3.8).
} 
Conditions (3.9) corresponds to consider confinement in a $p+1$ dimensional theory at finite temperature. Because we are interested in the $p$-dimensional theory at zero temperature, we should consider both directions among the $p$ ones. In this case we have (3.3) with the replacement of $a$ with $\tilde{a}$; the confinement condition (3.9) now reads,

$$
\gamma \equiv-\tilde{a}>0 \Longrightarrow u_{\min }=u_{0}\left(1+\frac{D-1}{2}|\tilde{a}|\right)^{\frac{1}{D-1}}
$$

For $\tilde{a}=0$, we get that $u_{d i v}=u_{0}$ gives also confinement, with a string tension,

$$
\sigma_{s}=T_{s} F\left(u_{d i v}\right)=T_{s} l_{0}^{2} u_{0}^{2}=\frac{D(D-1)}{2 \pi(10-D)} u_{0}^{2}
$$

This is the case analyzed by K-S. Finally, for $\tilde{a}>0$ there is no confinement.

It is important to note that, in contrast to what happens in critical superstring models, the string tension is given by the scale $u_{0}^{2}$ up to a numerical constant; therefore in the non-critical set-up, $u_{0}$ always results fixed to the typical scale of the theory,

$$
u_{0} \sim \Lambda_{Q C D}
$$

\section{The gauge-invariant perturbative setup}

According to the gauge/gravity correspondence, in order to determine the glue-ball mass spectra we must solve the linearized supergravity equations of motion in the background (2.2) [6], [7]. The fields present in the low energy effective actions are the metric $G$, the antisymmetric Kalb-Ramond tensor, the dilaton $\Phi$ and the $\mathrm{RR} q+1$-forms $A_{q+1}$, with the values of $q$ depending on the theory.

We will analyze consistent perturbations that leads to the equations to be considered in the next Sections. As noted in [19], in the string frame exists a complicated mixing between graviton perturbations and the rest. Fortunately as we will show, that does not occur in the Einstein frame for the family (2.2) in almost all the fluctuations; only the scalars from the metric perturbations lead to coupled systems. Then, since now on we switch to it in order to perform the computations, recalling that the metric tensors in both frames are related by $\left.G\right|_{\mathrm{s}-\mathrm{f}}=\left.e^{\frac{4}{D-2} \Phi} G\right|_{\mathrm{e}-\mathrm{f}}$.

The low energy effective action for non critical strings in Einstein frame is, ${ }^{2}$,

$S\left[G, \Phi, A_{q+1}\right]=\frac{1}{2 \kappa_{D}^{2}} \int \epsilon_{G}\left(R[G]-\frac{4}{D-2}(D(\Phi))^{2}+\Lambda^{2} e^{\frac{4}{D-2} \Phi}-\frac{1}{2} \sum_{q} e^{2 \alpha_{q} \Phi}\left(F_{q+2}\right)^{2}\right)$

${ }^{2}$ We use the compact notation,

$$
(\Omega \cdot \Lambda)_{A_{1} \ldots A_{p} ; B_{1} \ldots B_{p}} \equiv \frac{1}{q !} G^{C_{1} D_{1}} \ldots G^{C_{q} D_{q}} \Omega_{C_{1} \ldots C_{q} A_{1} \ldots A_{p}} \Lambda_{D_{1} \ldots D_{q} B_{1} \ldots B_{p}}
$$

where $\Omega$ and $\Lambda$ are arbitrary $(p+q)$-forms. 


$$
\alpha_{q}=\frac{D-2 q-4}{D-2}-0(1) \quad, \quad \text { RR (NSNS) forms }
$$

where $F_{q+2}=d A_{q+1}$ is the field strength of the gauge field form $A_{q+1}$, and the possible $q$ 's depend on the theory. The volume element is $\epsilon_{G}=\omega^{0} \wedge \cdots \wedge \omega^{D-1}=d^{D} x E$, with $\left\{\omega^{A}\right\}$ the vielbein.

The equations of motion that follows from (4.2) are,

$$
\begin{aligned}
R_{A B} & =\frac{4}{D-2} D_{A} \Phi D_{B} \Phi-\frac{\Lambda^{2}}{D-2} e^{\frac{4}{D-2} \Phi} G_{A B} \\
& +\frac{1}{2} \sum_{q} e^{2 \alpha_{q} \Phi}\left(\left(F_{q+2}\right)_{A ; B}^{2}-\frac{q+1}{D-2}\left(F_{q+2}\right)^{2} G_{A B}\right) \\
0 & =D^{2}(\Phi)+\frac{\Lambda^{2}}{2} e^{\frac{4}{D-2} \Phi}-\frac{D-2}{8} \sum_{q} \alpha_{q} e^{2 \alpha_{q} \Phi}\left(F_{q+2}\right)^{2} \\
d\left(e^{2 \alpha_{q} \Phi} * F_{q+2}\right) & =(-)^{q} Q_{q} * J_{q+1} \quad, \quad Q_{q} \equiv 2 \kappa_{D}^{2} \mu_{q}
\end{aligned}
$$

where we have introduced a current $J_{q+1}$ of $q$-brane source with tension $\mu_{q}$.

The perturbations around a classical solution $\left(G, \Phi, A_{q}\right)$ are written as,

$$
\text { metric } \rightarrow G_{A B}+h_{A B} \quad, \quad \text { dilaton } \rightarrow \Phi+\xi \quad, \quad \text { q-form } \rightarrow A_{q}+a_{q}
$$

The linear equations for the perturbations $\left(h, \xi, a_{q}\right)$ that follows from (4.3) are, $\underline{\text { h-equations }}$

$$
\begin{aligned}
0 & =A_{A B}(h)-\left(\frac{2 \Lambda^{2}}{D-2} e^{\frac{4}{D-2} \Phi}+\sum_{q} \frac{q+1}{D-2} e^{2 \alpha_{q} \Phi}\left(F_{q+2}\right)^{2}\right) h_{A B} \\
& +\sum_{q} e^{2 \alpha_{q} \Phi}\left(\left(-\left(F_{q+2}\right)^{2} C A ; D B\right.\right. \\
& \left.+\frac{q+1}{D-2} G_{A B}\left(F_{q+2}\right)^{2} C_{C ; D}\right) h^{C D} \\
& \left.+\left(F_{q+2} \cdot f_{q+2}\right)_{A ; B}+\left(F_{q+2} \cdot f_{q+2}\right)_{B ; A}-2 \frac{q+1}{D-2} G_{A B} F_{q+2} \cdot f_{q+2}\right) \\
& +\left(\sum_{q} 2 \alpha_{q} e^{2 \alpha_{q} \Phi}\left(\left(F_{q+2}\right)_{A ; B}^{2}-\frac{q+1}{D-2}\left(F_{q+2}\right)^{2} G_{A B}\right)-\frac{8 \Lambda^{2}}{(D-2)^{2}} e^{\frac{4}{D-2} \Phi} G_{A B}\right) \xi \\
& +\frac{8}{D-2}\left(D_{A} \Phi D_{B} \xi+D_{B} \Phi D_{A} \xi\right)
\end{aligned}
$$

where $A_{A B}(h)$ is worked out in Appendix A.

$\xi$-equation

$$
\begin{aligned}
0 & =D^{2}(\xi)+\left(\frac{2 \Lambda^{2}}{D-2} e^{\frac{4}{D-2} \Phi}-\frac{D-2}{4} \sum_{q} \alpha_{q}^{2} e^{2 \alpha_{q} \Phi}\left(F_{q+2}\right)^{2}\right) \xi \\
& +\left(-D_{A} D_{B}(\Phi)+\frac{D-2}{16} \sum_{q} \alpha_{q} e^{\alpha_{q} \Phi}\left(F_{q+2}\right)^{2}{ }_{A ; B}\right) h^{A B} \\
& -D^{C}(\Phi)\left(D^{D} h_{C D}-\frac{1}{2} D_{C} h_{D}^{D}\right)-\frac{D-2}{4} \sum_{q} \alpha_{q} e^{2 \alpha_{q} \Phi} F_{q+2} \cdot f_{q+2}
\end{aligned}
$$


$\underline{a_{q+1} \text {-equations }}$

$$
\begin{aligned}
& 0=-e^{-2 \alpha_{q} \Phi} D^{B}\left(e^{2 \alpha_{q} \Phi}\left(f_{q+2}\right)_{A_{1} \ldots A_{q+1} B}\right)-2 \alpha_{q} e^{-2 \alpha_{q} \Phi} D^{B}\left(e^{2 \alpha_{q} \Phi}\left(F_{q+2}\right)_{A_{1} \ldots A_{q+1} B} \xi\right) \\
& +e^{-\alpha_{q} \Phi} D^{B}\left(e^{\alpha_{q} \Phi}\left(F_{q+2}\right)_{A_{1} \ldots A_{q+1}{ }^{C}} h_{B C}\right)-\frac{1}{2}\left(F_{q+2}\right)_{A_{1} \ldots A_{q+1}}{ }^{B} D_{B} h_{C}^{C}
\end{aligned}
$$

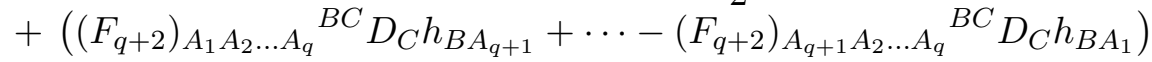

We remark that, for general backgrounds, the diagonalization is not possible. However, for the backgrounds (2.2) (and working in Einstein frame), we will see that it results rather simply.

The system of equations (4.3) is of course invariant under re-parameterizations, in particular under infinitesimal ones, $x^{M} \rightarrow x^{M}-\epsilon^{M}(x)+o\left(\epsilon^{2}\right)$, with the fields transforming as tensors. On the other hand, (4.5) can be seen as a system of equations for fields $\left(h, \xi, a_{q+1}\right)$ in a background $\left(G, \Phi, A_{q+1}\right)$. Diffeomorphism invariance translates as invariance under,

$$
h \rightarrow h+L_{\epsilon}(G) \quad, \quad \xi \rightarrow \xi+L_{\epsilon}(\Phi) \quad, \quad a_{q} \rightarrow a_{q}+L_{\epsilon}\left(A_{q}\right)
$$

where $L_{\epsilon}(\ldots)$ stands for the Lie derivative w.r.t. the vector field $\epsilon=\epsilon^{M}(x) \partial_{M}=\epsilon^{A}(x) e_{A}$. More explicitly, it is not difficult to show that (4.5)-(4.7) are left invariant under the field transformations ${ }^{3}$,

$$
\begin{aligned}
{ }^{\epsilon} h_{A B} & =h_{A B}+D_{A} \epsilon_{B}+D_{B} \epsilon_{A} \\
\epsilon_{\xi} & =\xi+\epsilon^{A} D_{A} \Phi \\
{ }^{\epsilon} a_{q A_{1} \ldots A_{q}} & =a_{q A_{1} \ldots A_{q}}+\epsilon^{B} D_{B} A_{q A_{1} \ldots A_{q}}+D_{A_{1}} \epsilon^{B} A_{q B A_{2} \ldots A_{q}}+\cdots+D_{A_{q}} \epsilon^{B} A_{q A_{1} \ldots A_{q-1} B}
\end{aligned}
$$

As the system is linear, it follows that $\left(L_{\epsilon}(G), L_{\epsilon}(\Phi), L_{\epsilon}\left(A_{q}\right)\right)$ is solution for any $\epsilon$, the pure gauge, trivial, solution. In contrast to ordinary gauge theories where the transformations are non linear, in the case at hand of linear perturbation theory it should be possible to define explicitly gauge invariant quantities, and to express the equations for the perturbations (4.5) in terms of them in a manifest gauge invariant way. It is worth to remark here that, since the pioneer work by J.M. Bardeen [32], gauge-invariant perturbation theory was developed in the last decades mainly in cosmological contexts ${ }^{4}$. For the family that will concern in this paper we can do it as follows. First, we introduce the fluctuation $\chi$ according to,

$$
f_{D} \equiv \chi F_{D} \quad, \quad{ }^{\epsilon} \chi=\chi+D_{A} \epsilon^{A}
$$

where the gauge transformation of $\chi$ follows from (4.9). Next we observe that,

$$
I_{\xi} \equiv \xi \quad, \quad I_{\chi} \equiv \chi-\frac{1}{2} h_{A}^{A}
$$

\footnotetext{
${ }^{3}$ This is a generalization of the usual perturbative treatment around flat space in the context of General Relativity, see for example [31], chapter 6.

${ }^{4}$ For the extension to second order perturbation theory, see 33 .
} 
are both gauge invariant, together with the $q+1$-form fields $a_{q+1}, q \neq D-2$. In terms of them, the fluctuation equations (4.5)-(4.7) are written in a manifest gauge invariant way,

$$
\begin{aligned}
0 & =A_{A B}(h)-\frac{2 \Lambda^{2}}{D} e^{\frac{4}{D-2} \Phi} h_{A B}-\frac{8 \Lambda^{2} e^{\frac{4}{D-2} \Phi}}{D(D-2)} G_{A B}\left(\frac{2 D}{D-2} I_{\xi}-I_{\chi}\right) \\
0 & =D^{2}\left(I_{\xi}\right)+\frac{D+2}{D-2} \Lambda^{2} e^{\frac{4}{D-2} \Phi} I_{\xi}-\Lambda^{2} e^{\frac{4}{D-2} \Phi} I_{\chi} \\
0 & =D^{B}\left(e^{2 \alpha_{q} \Phi}\left(f_{q+2}\right)_{A_{1} \ldots A_{q+1} B}\right) \quad, \quad q \neq D-2 \\
0 & =D_{A}\left(\frac{2 D}{D-2} I_{\xi}-I_{\chi}\right)
\end{aligned}
$$

From the last, $a_{D-1}$-equation, it follows the relation,

$$
I_{\chi}=\frac{2 D}{D-2} I_{\xi}
$$

Hence we get a partially decoupled system,

$$
\begin{aligned}
0 & =A_{A B}(h)-\frac{2 \Lambda^{2}}{D} e^{\frac{4}{D-2} \Phi} h_{A B} \\
0 & =D^{2}\left(I_{\xi}\right)-\frac{\Lambda^{2}}{D-2} e^{\frac{4}{D-2} \Phi} I_{\xi} \\
0 & =D^{B}\left(e^{2 \alpha_{q} \Phi}\left(f_{q+2}\right)_{A_{1} \ldots A_{q+1} B}\right) \quad, \quad q \neq D-2
\end{aligned}
$$

All the perturbative spectrum comes from these equations. It is worth to note that the metric equation is gauge invariant, as can be checked by using the general property,

$$
A_{A B}\left({ }^{\epsilon} h\right)-A_{A B}(h)=-2\left(D_{C} R_{A B} \epsilon^{C}+R_{A C} D_{B} \epsilon^{C}+R_{B C} D_{A} \epsilon^{C}\right)
$$

and the background field equations of motion (4.3). Later in Subsection 4.3 further gauge invariant fields will be constructed from the metric fluctuations, for the particular ansätz to be considered.

\subsection{The equation for dilatonic fluctuations}

They correspond to take ${ }^{5}$,

$$
h_{A B}=0 \quad ; \quad f_{q+2} \equiv d a_{q+1}= \begin{cases}0 & , q \neq D-2 \\ (-)^{p+1} \frac{2 D}{D-2} Q_{D-2} e^{\frac{2 D}{D-2} \Phi} \epsilon_{G} I_{\xi}, & q=D-2\end{cases}
$$

with $I_{\xi}$ satisfying the second equation in (4.14). As usual, we Fourier decompose the perturbation,

$$
I_{\xi}(x, u)=\chi(u) e^{i p_{a} x^{a}}
$$

From the translational symmetries in the $x^{a}$-coordinates of the background, the modes do not mix. In Section 5 we will consider some of them compactified.

\footnotetext{
${ }^{5}$ With the no gauge-invariant statement $h_{A B}=0$, we really mean that we put to zero the possible gauge invariant metric fluctuations constructed from $h_{A B}$, see subsection 4.3 .
} 
By introducing (4.17) in (4.14), $I_{\xi}$-equation, we get,

$$
\frac{1}{E} \partial_{u}\left(\frac{E}{C^{2}} \partial_{u} \chi(u)\right)-\left(\sum_{a} \frac{p^{a} p_{a}}{A_{a}^{2}}+\Lambda^{2}\right) \chi(u)=0
$$

where the metric functions are those in the string frame (2.2). The following change of variable and definition,

$$
\begin{aligned}
\left(\frac{u}{u_{0}}\right)^{D-1} & =1+e^{x} \equiv g(x) \quad, \quad x \in \Re \\
\chi(u) & \equiv g(x)^{-\frac{1}{2}} H(x)
\end{aligned}
$$

puts 4.18$)$ in the Schrödinger form for $H(x)$,

$$
0=-H^{\prime \prime}(x)+V(x) H(x)
$$

with the potential,

$$
V(x)=\frac{1}{4}-\frac{1}{4 g(x)^{2}}+\sum_{a} \hat{p}^{a} \hat{p}_{a} \frac{e^{\left(1-a_{a}\right) x}}{g(x)^{1-a_{a}+\frac{2}{D-1}}}+\frac{D}{D-1}\left(1+e^{-x}\right)^{-1}
$$

where $\hat{p}_{a} \equiv \frac{1}{(D-1) u_{0}} p_{a}$.

\subsection{The equation for RR one-form fluctuations}

Among the possible $(q+1)$-forms fluctuations, we will consider the $\mathrm{RR} a_{1}$ field that it is always present in D-dimensional type IIA NCST ${ }^{6}$. According to (4.14), the fluctuation obtained by switching on only $a_{q+1}$, for any $q \neq D-2$ is consistent if $a_{q+1}$ satisfy the generalized Maxwell equations in the background metric. In particular, for the $a_{1}$ form,

$$
D^{B}\left(e^{2 \alpha_{q} \Phi} D_{A}\left(a_{1 B}\right)\right)-D^{B}\left(e^{2 \alpha_{q} \Phi} D_{B}\left(a_{1 A}\right)\right)=0
$$

The general Fourier form is,

$$
a_{A}(x, u)=\chi_{A}(u) e^{i p_{a} x^{a}}
$$

By using the results collected in Appendix A we get,

$$
\begin{aligned}
& D^{B} F_{a B} e^{-i p_{c} x^{c}}=\sum_{c} \frac{p^{c} p_{c}}{A_{c}^{2}} P_{a}^{b} \chi_{b}+\frac{C A_{a}}{E} e_{n}\left(\frac{E}{C A_{a}}\left(i \frac{p^{a}}{A_{a}} \chi_{n}-e_{n}\left(\chi_{a}\right)-\sigma_{a} \chi_{a}\right)\right) \\
& D^{B} F_{n B} e^{-i p_{c} x^{c}}=\sum_{b} \frac{p^{b} p_{b}}{A_{b}{ }^{2}} \chi_{n}+i \sum_{b} \frac{p^{b}}{A_{b}}\left(e_{n}\left(\chi_{b}\right)+\sigma_{b} \chi_{b}\right)
\end{aligned}
$$

where $P_{a}{ }^{b} \equiv \delta_{a}{ }^{b}-\left(\sum_{c} \frac{p^{c} p_{c}}{A_{c}{ }^{2}}\right)^{-1} \frac{p_{a}}{A_{a}} \frac{p^{b}}{A_{b}}$. The second equation (in the u-polarization) is just a constraint that gives $\chi_{n}$ in terms of the $\chi_{b}$ 's. By plugging it in the first equation we get,

$$
\frac{C A_{a}}{E} e_{n}\left(\frac{E}{C A_{a}} P_{a}^{b}\left(e_{n}\left(\chi_{b}\right)+\sigma_{b} \chi_{b}\right)\right)-\sum_{c} \frac{p^{c} p_{c}}{A_{c}^{2}} P_{a}^{b} \chi_{b}=0
$$

We will reduce this coupled system in the next section by using standard ansätz in each of the models to be considered.

\footnotetext{
${ }^{6}$ In $D=8$ it is also present $a_{3}$, and of course the Kalb-Ramond field $B_{2} A B$ from the NS-NS sector in any dimension, but they will not be considered in this paper.
} 


\subsection{The equation for the metric fluctuations}

From (4.14),

$$
I_{\xi}=I_{\chi}=0 \quad ; \quad f_{q+2} \equiv d a_{q+1}= \begin{cases}0 & , q \neq D-2 \\ \frac{(-)^{D}}{2} Q_{D-2} e^{\frac{2 D}{D-2} \Phi} \epsilon_{G} h_{C}^{C}, & q=D-2\end{cases}
$$

is consistent if the metric perturbation satisfy,

$$
A_{A B}(h) \equiv D_{A} D_{B} h_{C}^{C}+D^{2} h_{A B}-D^{C} D_{A} h_{C B}-D^{C} D_{B} h_{A C}=\frac{2 \Lambda^{2}}{D} e^{\frac{4}{D-2} \Phi} h_{A B}
$$

In order to write it in manifest gauge invariant way, we introduce the fields $\left(g, g_{a}, I_{a b}\right)$ by,

$$
\begin{aligned}
e_{n}(g) & \equiv h_{n n} \\
A_{a} e_{n}\left(\frac{g_{a}}{A_{a}}\right) & \equiv h_{a n}-\frac{1}{2} e_{a}(g) \\
I_{a b} & \equiv h_{a b}-e_{a}\left(g_{b}\right)-e_{b}\left(g_{a}\right)-\eta_{a b} \sigma_{a} g
\end{aligned}
$$

that under gauge transformations go to,

$$
\delta_{\epsilon} g=2 \epsilon_{n} \quad, \quad \delta_{\epsilon} g_{a}=\epsilon_{a} \quad, \quad \delta_{\epsilon} I_{a b}=0
$$

The equations that follow from (4.27) and (A.10) are,

$$
\begin{aligned}
0 & =e^{A} e_{A}\left(I_{a b}\right)+e_{a} e_{b}\left(I_{c}^{c}\right)-e^{c} e_{a}\left(I_{b c}\right)-e^{c} e_{b}\left(I_{a c}\right)+\sigma e_{n}\left(I_{a b}\right)-\left(\sigma_{a}-\sigma_{b}\right)^{2} I_{a b} \\
& +\eta_{a b} \sigma_{a} e_{n}\left(I_{c}^{c}\right) \\
0 & =e^{c} e_{n}\left(I_{a c}\right)-e_{a} e_{n}\left(I_{c}^{c}\right)+\left(\sigma_{c}-\sigma_{a}\right)\left(e^{c}\left(I_{a c}\right)-e_{a}\left(I_{c}^{c}\right)\right) \\
0 & =e_{n}^{2}\left(I_{c}^{c}\right)+2 \sigma_{c} e_{n}\left(I_{c}^{c}\right)
\end{aligned}
$$

where the sum over the " $c$ " index is understood in the last two equations. The dependence on $g$ and $g_{a}$ has fallen down leaving all the equations expressed in terms of the gauge invariant fluctuation fields $I_{a b}$. The Fourier modes are introduced as usual,

$$
I_{a b}(x, u)=\chi_{a b}(u) e^{i p_{a} x^{a}}
$$

The equations for $\chi_{A B}(u)$ that follow from (4.30) results,

$$
\begin{aligned}
0 & =e_{n}^{2}\left(\chi_{a b}\right)+\sigma e_{n}\left(\chi_{a b}\right)+\eta_{a b} \sigma_{a} e_{n}\left(\chi_{c}^{c}\right)-\frac{p_{a} p_{b}}{A_{a} A_{b}} \chi_{c}^{c}+\left(-\frac{p^{c} p_{c}}{A_{c}^{2}}-\left(\sigma_{a}-\sigma_{b}\right)^{2}\right) \chi_{a b} \\
& +\frac{p_{a}}{A_{a}} \frac{p^{c}}{A_{c}} \chi_{b c}+\frac{p_{b}}{A_{b}} \frac{p^{c}}{A_{c}} \chi_{a c} \\
0 & =\frac{p^{c}}{A_{c}} e_{n}\left(\chi_{a c}\right)-\frac{p_{a}}{A_{a}} e_{n}\left(\chi_{c}^{c}\right)+\left(\sigma_{c}-\sigma_{a}\right)\left(\frac{p^{c}}{A_{c}} \chi_{a c}-\frac{p_{a}}{A_{a}} \chi_{c}^{c}\right) \\
0 & =\sum_{c} \frac{1}{A_{c}^{2}} e_{n}\left(A_{c}^{2} e_{n}\left(\chi_{c}^{c}\right)\right)
\end{aligned}
$$




\section{Holographic models of $d$ dimensional Yang-Mills theories}

Let us take, among the $x^{a}$ coordinates, $d$ non compact, equivalent, $x^{\mu}$-coordinates, $\mu=$ $0,1, \ldots, d-1$, and $D-d-1$ compact and non equivalent $\tau^{i}, i=1, \ldots D-d-1, \tau_{i} \equiv$ $\tau_{i}+2 \pi R_{i}$. We will denote with a $\sim$ quantities associated with the non-compact directions $\left(A_{\mu}=\tilde{A}, a_{\mu} \tilde{a}, \sigma_{\mu}=\tilde{\sigma}\right.$, etc). The metric and constraints (2.3) are,

$$
\begin{aligned}
l_{0}^{-2} G= & u^{2}\left(f(u)^{\tilde{a}} \eta_{\mu \nu} d x^{\mu} d x^{\nu}+\sum_{i} f(u)^{a_{i}} d \tau^{i 2}\right)+\frac{d u^{2}}{u^{2} f(u)} \\
& d \tilde{a}+\sum_{i} a_{i}=1 \quad, \quad d \tilde{a}^{2}+\sum_{i} a_{i}{ }^{2}=1
\end{aligned}
$$

We stress that $D-d-2$ exponents remain free.

\subsection{Dilatonic fluctuations}

From (4.21), the equation to solve is,

$$
\begin{aligned}
0 & =-H^{\prime \prime}(x)+V(x) H(x) \\
V(x) & =\frac{1}{4}-\frac{1}{4 g(x)^{2}}+\frac{D}{D-1} \frac{e^{x}}{g(x)}-\hat{M}^{2} \frac{e^{(1-\tilde{a}) x}}{g(x)^{1-\tilde{a}+\frac{2}{D-1}}}+\sum_{i} \hat{p}_{i}{ }^{2} \frac{e^{\left(1-a_{i}\right) x}}{g(x)^{1-a_{i}+\frac{2}{D-1}}}
\end{aligned}
$$

where $M \equiv(D-1) u_{0} \hat{M}$ is the $d$-dimensional mass. The terms with momentum in the compact directions are quantized in units of $R_{i}{ }^{-1}$, and represent Kaluza-Klein modes, and decouple for $R_{i} \rightarrow 0$;

\subsection{RR gauge field: transverse fluctuations}

The consistent ansätz includes the transverse condition,

$$
\chi_{\mu}(u)=\epsilon_{\mu}(p) \chi(u) \quad ; \quad \epsilon_{\mu}(p) p^{\mu}=0
$$

From (4.25), (4.19), we obtain,

$$
\begin{aligned}
0 & =-H^{\prime \prime}(x)+V(x) H(x) \\
V(x) & =\frac{1}{4 g(x)^{2}}\left(\left(\frac{D-3}{D-1} e^{x}-\tilde{a}\right)^{2}+2\left(\frac{D-3}{D-1}+\tilde{a}\right) e^{x}\right)-\hat{M}^{2} \frac{e^{(1-\tilde{a}) x}}{g(x)^{1-\tilde{a}+\frac{2}{D-1}}} \\
& +\sum_{i} \hat{p}_{i}{ }^{2} \frac{e^{\left(1-a_{i}\right) x}}{g(x)^{1-a_{i}+\frac{2}{D-1}}}
\end{aligned}
$$

\subsection{RR gauge field: longitudinal fluctuations}

The consistent ansätz is, at fixed $i$ (but for any $i=1, \ldots, D-d-1$ ),

$$
\chi_{i}(u)=\chi(u) \quad ; \quad p_{i}=0
$$

From (4.25), (4.19), we get,

$$
0=-H^{\prime \prime}(x)+V(x) H(x)
$$




$$
\begin{aligned}
V(x) & =\frac{1}{4 g(x)^{2}}\left(\left(\frac{D-3}{D-1} e^{x}-a_{i}\right)^{2}+2\left(\frac{D-3}{D-1}+a_{i}\right) e^{x}\right)-\hat{M}^{2} \frac{e^{(1-\tilde{a}) x}}{g(x)^{1-\tilde{a}+\frac{2}{D-1}}} \\
& +\sum_{j \neq i} \hat{p}_{j}{ }^{2} \frac{e^{\left(1-a_{j}\right) x}}{g(x)^{1-a_{j}+\frac{2}{D-1}}}
\end{aligned}
$$

\subsection{Metric: transverse fluctuations}

They correspond to take the ansätz,

$$
\chi_{\mu \nu}(u)=\epsilon_{\mu \nu}(p) \chi(u) \quad ; \quad \epsilon_{\rho}^{\rho}=0, \epsilon_{\mu \nu} p^{\nu}=0
$$

and the rest zero. Equations (4.32) are satisfied if, after making the change in (4.19), $H$ obeys the equation,

$$
\begin{aligned}
0 & =-H^{\prime \prime}(x)+V(x) H(x) \\
V(x) & =\frac{1}{4}-\frac{1}{4 g(x)^{2}}-\hat{M}^{2} \frac{e^{(1-\tilde{a}) x}}{g(x)^{1-\tilde{a}+\frac{2}{D-1}}}+\sum_{i} \hat{p}_{i}{ }^{2} \frac{e^{\left(1-a_{i}\right) x}}{g(x)^{1-a_{i}+\frac{2}{D-1}}}
\end{aligned}
$$

\subsection{Metric: longitudinal fluctuations}

They correspond to take the ansätz, at fixed $i$ (but for any $i$ ),

$$
\chi_{i \mu}(u)=\epsilon_{\mu}(p) \chi(u) \quad ; \quad p_{i}=0, \epsilon_{\mu} p^{\mu}=0
$$

and the rest zero. It obeys the equations (4.32) if, after making the change in (4.19), $\mathrm{H}$ satisfy the equation,

$$
\begin{aligned}
0 & =-H^{\prime \prime}(x)+V(x) H(x) \\
V(x) & =\frac{1}{4}-\frac{1-\left(\tilde{a}-a_{i}\right)^{2}}{4 g(x)^{2}}-\hat{M}^{2} \frac{e^{(1-\tilde{a}) x}}{g(x)^{1-\tilde{a}+\frac{2}{D-1}}}+\sum_{j \neq i} \hat{p}_{j}{ }^{2} \frac{e^{\left(1-a_{j}\right) x}}{g(x)^{1-a_{j}+\frac{2}{D-1}}}
\end{aligned}
$$

\subsection{Metric: scalar fluctuations}

This is the more complicated case, because it involves in general a coupled system. Only in the case analyzed in [34], that we review below, the system can be reduced to one equation of the type (4.21) for some potential, as it happened with the perturbations analyzed so far. So we think it is worth to present a somewhat detailed analysis of this case.

Let us consider the ansätz,

$$
\chi_{\mu \nu}(u)=a(u) \eta_{\mu \nu}+b(u) p_{\mu} p_{\nu} \quad, \quad \chi_{i j}(u)=a_{i}(u) \delta_{i j} \quad, \quad \chi_{\mu i}(u)=0
$$

We note that this ansätz depends on $D-d+1$ invariant functions $\left(a, a_{i}, b\right)$. We would like to stress that, at difference of [34], these functions are gauge invariant, and then all of them are relevant. It results convenient to introduce the following invariant fluctuations,

$$
\begin{aligned}
F & =a-\tilde{\sigma} \tilde{A}^{2} e_{n}(b) \\
F_{i} & =a_{i}-\sigma_{i} \tilde{A}^{2} e_{n}(b) \\
F_{n} & =-e_{n}\left(\tilde{A}^{2} e_{n}(b)\right)
\end{aligned}
$$


In terms of them, equations (4.32) are written as,

$$
\begin{aligned}
& 0=e_{n}{ }^{2}(F)+\sigma e_{n}(F)+\tilde{\sigma} e_{n}\left(d F+F_{\tau}-F_{n}\right)+\frac{M^{2}}{\tilde{A}^{2}} F-\frac{2 \Lambda^{2}}{D} e^{\frac{4}{D-2} \Phi} F_{n} \\
& 0=e_{n}{ }^{2}\left(F_{i}\right)+\sigma e_{n}\left(F_{i}\right)+\sigma_{i} e_{n}\left(d F+F_{\tau}-F_{n}\right)+\frac{M^{2}}{\tilde{A}^{2}} F_{i}-\frac{2 \Lambda^{2}}{D} e^{\frac{4}{D-2} \Phi} F_{n} \\
& 0=e_{n}{ }^{2}\left(d F+F_{\tau}\right)+2 d \tilde{\sigma} e_{n}(F)+2 \sum_{i} \sigma_{i} e_{n}\left(F_{i}\right)-\sigma e_{n}\left(F_{n}\right)+\left(\frac{M^{2}}{\tilde{A}^{2}}-\frac{2 \Lambda^{2}}{D} e^{\frac{4}{D-2} \Phi}\right) F_{n} \\
& 0=e_{n}\left((d-1) F+F_{\tau}\right)+\sum_{i}\left(\sigma_{i}-\tilde{\sigma}\right) F_{i}-(\sigma-\tilde{\sigma}) F_{n} \\
& 0=(d-2) F+F_{\tau}+F_{n}
\end{aligned}
$$

where $F_{\tau} \equiv \sum_{i} F_{i}$. The last equation clearly is a constraint, that we trivially solve for $F_{n}=-(d-2) F-F_{\tau}$. The remaining equations take the form,

$$
\begin{aligned}
0 & =e_{n}^{2}(F)+\sigma e_{n}(F)+2 \tilde{\sigma} e_{n}\left((d-1) F+F_{\tau}\right)+\frac{M^{2}}{\tilde{A}^{2}} F+\frac{2 \Lambda^{2}}{D} e^{\frac{4}{D-2} \Phi}\left((d-2) F+F_{\tau}\right) \\
0 & =e_{n}^{2}\left(F_{i}\right)+\sigma e_{n}\left(F_{i}\right)+2 \sigma_{i} e_{n}\left((d-1) F+F_{\tau}\right)+\frac{M^{2}}{\tilde{A}^{2}} F_{i}+\frac{2 \Lambda^{2}}{D} e^{\frac{4}{D-2} \Phi}\left((d-2) F+F_{\tau}\right) \\
0 & =e_{n}^{2}\left(d F+F_{\tau}\right)+\sigma e_{n}\left(d F+F_{\tau}\right)+2 \sum_{i} \sigma_{i} e_{n}\left(F_{i}-F\right) \\
& +\left(-\frac{M^{2}}{\tilde{A}^{2}}+\frac{2 \Lambda^{2}}{D} e^{\frac{4}{D-2} \Phi}\right)\left((d-2) F+F_{\tau}\right) \\
0 & =e_{n}\left((d-1) F+F_{\tau}\right)+(d-2)(\sigma-\tilde{\sigma}) F+\sum_{i}\left(\sigma_{i}+\sigma-2 \tilde{\sigma}\right) F_{i}
\end{aligned}
$$

At this point we note three facts,

- There are $D-d$ unknowns $\left(F, F_{i}\right)$ and $D-d+2$ differential equations; this is obviously related with the fixed gauge invariance;

- The $e_{n}\left((d-1) F+F_{\tau}\right)$ terms in the first two equations in 5.13) can be eliminated by using the last one;

- The third equation can be transformed in a first order one by using the first two equations.

By taking into account all these facts, the system (5.13) can be partitioned in two sets, a second order system of $D-d$ equations with $D-d$ unknowns,

$$
\begin{aligned}
0 & =e_{n}^{2}(F)+\sigma e_{n}(F)+\left(\frac{M^{2}}{\tilde{A}^{2}}+2(d-2)\left(\frac{\Lambda^{2}}{D} e^{\frac{4}{D-2} \Phi}-\tilde{\sigma}(\sigma-\tilde{\sigma})\right)\right) F \\
& +2 \sum_{i}\left(\frac{\Lambda^{2}}{D} e^{\frac{4}{D-2} \Phi}-\tilde{\sigma}\left(\sigma_{i}+\sigma-2 \tilde{\sigma}\right)\right) F_{i} \\
0 & =e_{n}^{2}\left(F_{i}\right)+\sigma e_{n}\left(F_{i}\right)+2(d-2)\left(\frac{\Lambda^{2}}{D} e^{\frac{4}{D-2} \Phi}-\sigma_{i}(\sigma-\tilde{\sigma})\right) F \\
& +\sum_{j}\left(\frac{M^{2}}{\tilde{A}^{2}} \delta_{i j}+\frac{2 \Lambda^{2}}{D} e^{\frac{4}{D-2} \Phi}-2 \sigma_{i}\left(\sigma_{j}+\sigma-2 \tilde{\sigma}\right)\right) F_{j}
\end{aligned}
$$


and two first order equations,

$$
\begin{aligned}
0 & =\sum_{i} \sigma_{i} e_{n}\left(F-F_{i}\right)+\left((d-1) \frac{M^{2}}{\tilde{A}^{2}}+(d-2) \sum_{i} \sigma_{i}\left(\tilde{\sigma}-\sigma_{i}\right)\right) F \\
& +\sum_{i}\left(\frac{M^{2}}{\tilde{A}^{2}}+\sum_{j} \sigma_{j}\left(\tilde{\sigma}-\sigma_{j}\right)+\sigma\left(\tilde{\sigma}-\sigma_{i}\right)\right) F_{i} \\
0 & =e_{n}\left((d-1) F+F_{\tau}\right)+(d-2)(\sigma-\tilde{\sigma}) F+\sum_{i}\left(\sigma_{i}+\sigma-2 \tilde{\sigma}\right) F_{i}
\end{aligned}
$$

Let us first concentrate on the second order system (5.15). According to (4.19), we introduce the variable $x$ and the fields $\left(H, H_{i}\right)$ by,

$$
F(u) \equiv g(x)^{-\frac{1}{2}} H(x) \quad, \quad F_{i}(u) \equiv g(x)^{-\frac{1}{2}} H_{i}(x)
$$

After some computations, (5.15) can be put in the form,

$$
\begin{aligned}
\overrightarrow{0} & =-\vec{H}^{\prime \prime}(x)+\mathbf{V}(x) \vec{H}(x) \\
\mathbf{V}(x) & \equiv v(x) \mathbf{1}+\left(\begin{array}{cc}
m(x) & \vec{m}^{(1) t}(x) \\
\vec{m}^{(2)}(x) & \mathbf{m}(x)
\end{array}\right)
\end{aligned}
$$

where $\vec{H}(x) \equiv\left(H(x), H_{1}(x), \ldots, H_{D-d-1}(x)\right)$. The elements that define the potential matrix $\mathbf{V}(x)$ are given by,

$$
\begin{aligned}
v(x) & =\frac{1}{4}-\frac{1}{4 g(x)^{2}}-\hat{M}^{2} \frac{e^{(1-\tilde{a}) x}}{g(x)^{1-\tilde{a}+\frac{2}{D-1}}} \\
m(x) & =\frac{D-4}{g(x)^{2}}\left(\frac{\tilde{a}}{2}(1-\tilde{a})+\frac{(D-3) \tilde{a}-1}{D-1} e^{x}-\frac{2}{(D-1)^{2}} e^{2 x}\right) \\
m_{i}^{(1)}(x) & =\frac{1}{g(x)^{2}}\left(\frac{\tilde{a}}{2}\left(1+a_{i}-2 \tilde{a}\right)+\frac{(D-4) \tilde{a}+a_{i}-1}{D-1} e^{x}-\frac{2}{(D-1)^{2}} e^{2 x}\right) \\
m_{i}^{(2)}(x) & =\frac{D-4}{g(x)^{2}}\left(\frac{a_{i}}{2}(1-\tilde{a})+\frac{(D-2) a_{i}-\tilde{a}-1}{D-1} e^{x}-\frac{2}{(D-1)^{2}} e^{2 x}\right) \\
\mathbf{m}_{i j}(x) & =\frac{1}{g(x)^{2}}\left(\frac{a_{i}}{2}\left(1+a_{j}-2 \tilde{a}\right)+\frac{(D-2) a_{i}+a_{j}-2 \tilde{a}-1}{D-1} e^{x}-\frac{2}{(D-1)^{2}} e^{2 x}\right)
\end{aligned}
$$

This is the system to be analyzed thorough in the computation of the respective spectra.

The case $d=D-2$

What about the linear equations (5.16)? For $d=D-2$ there is just one compact dimension $\tau^{i} \equiv \tau$, and consequently we introduce $a_{i} \equiv a_{\tau}$, etc. There exist two solutions in this case, corresponding to the values of the exponents given by $\left(\tilde{a}=0, a_{\tau}=1\right)$, and $\left(\tilde{a}=\frac{2}{D-1}, a_{\tau}=-\frac{D-3}{D-1}\right)$. The first one is just the AdS Schwarzchild black hole in $D$ dimensions; the period of $\tau$ is usually fixed as in (2.7) requiring absence of a conical singularity in the plane $\tau-u$ and is regular, while the second one is not regular anyway in the IR, and what is more important for us, it is not confining solution according to (3.11) 
and will not be considered. What this case has of particular is that, having two unknowns $F$ and $F_{\tau}$, the equations (5.16) constitute themselves a system of two first order equations with two unknowns. After the change (5.17), (5.16) becomes,

$$
\begin{array}{r}
0=\left(\begin{array}{c}
H(x) \\
H_{\tau}(x)
\end{array}\right)^{\prime}-\mathbf{U}(x)\left(\begin{array}{c}
H(x) \\
H_{\tau}(x)
\end{array}\right) \\
\mathbf{U}(x) \equiv \frac{e^{x}}{2 g(x)} 1+\left(\begin{array}{ll}
u_{11}(x) & u_{12}(x) \\
u_{21}(x) & u_{22}(x)
\end{array}\right)
\end{array}
$$

where the elements that define $\mathbf{U}(x)$ are given by,

$$
\begin{aligned}
u_{11} & =-\frac{(D-3)(D-1)}{D-2} \hat{M}^{2} \frac{e^{(1-\tilde{a}) x}}{g(x)^{1-\tilde{a}+\frac{2}{D-1}}} \frac{g(x)}{\frac{D-1}{2} a_{\tau}+e^{x}}-\frac{D-4}{D-1} \frac{\frac{D-1}{2} \tilde{a}+e^{x}}{g(x)} \\
u_{12} & =-\frac{D-1}{D-2} \hat{M}^{2} \frac{e^{(1-\tilde{a}) x}}{g(x)^{1-\tilde{a}+\frac{2}{D-1}}} \frac{g(x)}{\frac{D-1}{2} a_{\tau}+e^{x}}-\frac{1}{D-1} \frac{\left(\frac{D-1}{2} \tilde{a}+e^{x}\right)^{2}}{g(x)\left(\frac{D-1}{2} a_{\tau}+e^{x}\right)} \\
u_{21} & =\frac{(D-3)^{2}(D-1)}{D-2} \hat{M}^{2} \frac{e^{(1-\tilde{a}) x}}{g(x)^{1-\tilde{a}+\frac{2}{D-1}}} \frac{g(x)}{\frac{D-1}{2} a_{\tau}+e^{x}}-\frac{D-4}{D-1} \frac{\frac{D-1}{2} a_{\tau}+e^{x}}{g(x)} \\
u_{22} & =\frac{(D-3)(D-1)}{D-2} \hat{M}^{2} \frac{e^{(1-\tilde{a}) x}}{g(x)^{1-\tilde{a}+\frac{2}{D-1}}} \frac{g(x)}{\frac{D-1}{2} a_{\tau}+e^{x}} \\
& +\frac{1}{g(x)}\left(\frac{D-3}{D-1} \frac{\left(\frac{D-1}{2} \tilde{a}+e^{x}\right)^{2}}{\frac{D-1}{2} a_{\tau}+e^{x}}+\tilde{a}-\frac{1+a_{\tau}}{2}-\frac{D-2}{D-1} e^{x}\right)
\end{aligned}
$$

Now, it is easy to prove that (5.20) implies the second order system (5.18) if and only if the identity,

$$
\mathbf{V}(x)=\mathbf{U}^{\prime}(x)+\mathbf{U}(x)^{2}
$$

holds. We have verified this relation of compatibility by direct computation. Moreover, the equivalence of the whole set of equations to the linear system (5.20) also allows to attack the problem by just solving one second order equation in, for example, the field $H$, obtained by plugging in the second equation of $(5.20)$ the value of $H_{\tau}$ obtained from the first one in terms of $H$ and $H^{\prime}$. In the general case $D-d-1>1$, the linear equations acts presumably as constraints, and we must solve (5.18) and verify a posteriori (5.20). ${ }^{7} \mathrm{We}$ will follow this strategy in the next section, presenting also results related to this particular case, compatible with those found in 34.

\section{Glue-ball spectra of $3 D$ Yang-Mills theories.}

We will consider in this section non critical $I I A$ superstrings in $D=6$ dimensions. The RR forms present are $A_{1}$ (with $A_{3}$ as its Hodge dual) and $A_{5}$, with field strengths $F_{2}=d A_{1}$

\footnotetext{
${ }^{7}$ Conversely, if as we will do, we assume that (5.18) holds, then if we find a matrix $\mathbf{U}$ that verify 5.22 , it follows that,

$$
\left(\vec{H}^{\prime}(x)-\mathbf{U}(x) \vec{H}(x)\right)^{\prime}+\mathbf{U}(x)\left(\vec{H}^{\prime}(x)-\mathbf{U}(x) \vec{H}(x)\right)=0
$$

We believe that with a convenient choice of $\mathbf{U}$ (and maybe, determined boundary conditions), $\vec{H}^{\prime}(x)=$ $\mathbf{U}(x) \vec{H}(x)$, thing that certainly happens for $d=D-2$. This would leave us with a $(D-d)$-dimensional linear system that presumably implies the two equations (5.18), but we have not verified this due to the non triviality of 5.22
} 
and $F_{6}=d A_{5}$ respectively. Furthermore, we will take $d=3$ equivalent directions, and $D-d-1=2$ as compact and non equivalent. The family to consider is interpreted as solutions of $D 4$-branes wrapped on a two-torus of radius $\left(R_{1}, R_{2}\right)$. The constraint equations (2.3) are,

$$
3 \tilde{a}+a_{1}+a_{2}=1 \quad, \quad 3 \tilde{a}^{2}+a_{1}^{2}+a_{2}^{2}=1
$$

As remarked before, we like to study the dependence of spectra on the exponents. It is useful to solve the constraints in the form,

$$
\begin{aligned}
\sqrt{3} \tilde{a} & =\frac{\cos \beta^{-}-\cos \beta^{+}}{1-\cos \beta^{+} \cos \beta^{-}} \sin \beta^{-} \\
a_{1} & =\frac{\cos \beta^{-}-\cos \beta^{+}}{1-\cos \beta^{+} \cos \beta^{-}} \cos \beta^{-} \\
a_{2} & =-\frac{\sin \beta^{+} \sin \beta^{-}}{1-\cos \beta^{+} \cos \beta^{-}}
\end{aligned}
$$

where the space of solutions is an $S^{1}$ parameterized by $\beta \sim \beta+\pi$, and

$$
\beta^{ \pm} \equiv \beta \pm \beta_{0} \quad, \quad \tan \beta_{0} \equiv \frac{1}{\sqrt{d}}, \beta_{0} \in\left[0, \frac{\pi}{2}\right]
$$

For $\beta=\beta_{0}$ we have the KS solution; we can think of the family like a deformation of it with parameter $\beta^{-}=\beta-\beta_{0}$. Thought in this way, it appears natural to impose the periodicity condition (2.7) on at least one of the periodic variables [18] [21], thinking about it as the one that breaks SUSY. We remember that (2.7) comes from imposing a smoothness condition at $u=u_{0}$; however, it is not clear to us why should be correct to do so, even less to imposing on both of the compact coordinates, because our family is singular anyway there, so we will leave both radii free in the meantime.

Before presenting the numerical ${ }^{8}$ results we have obtained, it seems to us very instructive to see how a possible decoupling limit is at work, following standard analysis. First, the decoupling of the tower of open string states imposes a low energy limit, $l_{s} \equiv \sqrt{\alpha^{\prime}} \rightarrow 0$. Being the six-dimensional Newton constant $2 \kappa_{6}{ }^{2} \sim l_{s}^{4} g_{s}{ }^{9}$, this limit, at fixed $g_{s}$, also decouples bulk-open and bulk-bulk interactions. On the other hand, according to (2.8) the large $N$ limit leaves us with classical string theory. The question is posed in what remains on the world-volume of the $D 4$-brane.

We recall in first term that a non-critical $I I A$ vacuum (linear dilaton, cigar, etc.) preserves $2^{\frac{D}{2}}=8$ supercharges [16]. A BPS $D p$-brane merged on it usually preserves onehalf of the supercharges. In fact, it was showed in references [23, 24] that the low energy limit of a $D 3$-brane in the cigar vacuum is $\mathcal{N}=1$ super Yang Mills in four dimensions, i.e., it preserves $4=\frac{1}{2} 8$ supercharges. Now, our dilaton constant $D 4$ solution can be though

\footnotetext{
${ }^{8}$ The spectra was calculated both in the WKB approach as Numerically, but because of the great agreement between both, we only show in the tables Numerical computations.

${ }^{9}$ The numerical factor in critical type II theories is $(2 \pi)^{7}$; from, for example, the four-graviton scattering amplitude, it should be possible to fix it also in non critical theories, but to our knowledge this calculation (or any other that permits to fix the coupling) was not carried out. A similar remark can be made w.r.t. equation $6.4 ; \frac{T_{s}{ }^{2}}{T_{D p}}=(2 \pi)^{p-2} l_{s}^{p-3} g_{s}$ in critical ST; in NCST a computation of the exchange interaction between $D p$ - branes as the one sketched in chapter 13 of 36 .
} 
as the black version of a BPS $\left(u_{0}=0\right) D 4$ in the near horizon limit, that is T-dual to a BPS D3 brane living in the linear dilaton vacuum, which is the large $u$ limit of the cigar vacuum. So we could argue that our family describes in the UV some (unknown) CFT in five dimensions, the completion of the five dimensional YM theory whose coupling constant at scale $\Lambda_{s} \equiv l_{s}^{-1}$ is,

$$
g_{Y M_{5}}^{2}=\frac{T_{s}^{2}}{T_{D 4}} \sim l_{s} g_{s}
$$

The t'Hooft coupling at such scale, and the dimensionless effective coupling constant at scale $E$ are,

$$
\lambda_{5}{ }^{2} \equiv g_{Y M_{5}}^{2} N \sim l_{s} \quad ; \quad \lambda_{5}^{e f f}(E)^{2} \equiv \lambda_{5}^{2} E \sim \frac{E}{\Lambda_{s}}
$$

where we have used (2.8). From (6.5) two well-known facts follow; for $E<<\Lambda_{s}, \lambda_{5}^{\text {eff }}<<1$ and perturbative YM theory is valid at low energies. On the other hand it is clear that $g_{Y M_{5}}$ can not be fixed for $l_{s} \rightarrow 0$, and therefore no decoupling limit exists; this fact can be interpreted as a manifestation of the non-renormalizability of YM theories in dimensions higher that four [37]. However we are interested in the three-dimensional theory that we get below the compactification scale $\Lambda_{c} \equiv\left(4 \pi^{2} R_{1} R_{2}\right)^{-\frac{1}{2}}$; the t'Hooft coupling at such scale is,

$$
\lambda_{3}^{2}=\frac{\lambda_{5}^{2}}{4 \pi^{2} R_{1} R_{2}} \sim \frac{\Lambda_{c}^{2}}{\Lambda_{s}}
$$

Then, following Witten's argument [6], the compactification should break supersymmetry, giving masses to both fermions and bosons at tree and one-loop level respectively, the large compactification scale limit should decouple them, and three-dimensional YM should be reached in the limit 酒,

$$
\lambda_{3}^{2} \underset{\Lambda_{s} \rightarrow \infty}{\stackrel{\Lambda_{c} \rightarrow \infty}{\longrightarrow}} \Lambda_{Q C D} \sim u_{0} \quad \longleftrightarrow \quad \frac{\Lambda_{c}{ }^{2}}{\Lambda_{s}} \sim u_{0}
$$

where (3.13) was taken into account.

We will use the usual notation that assigns for every kind of perturbation the corresponding dual glueball notation $J^{P C}$. The spin $J$, parity $P$ and charge conjugation $C$, are deduced from the quantum numbers of the boundary operator that couples to the perturbation under consideration, we refer the reader to the literature [8, 13]

In the following sections we compute the spectra corresponding to every kind of perturbation.

\subsection{Spectrum from dilatonic fluctuations.}

The Schröedinger like equation to solve for zero energy corresponds to the potential,

$$
V(x)=\frac{1}{4}-\frac{1}{4 g(x)^{2}}+\frac{6}{5} \frac{e^{x}}{g(x)}-\hat{M}^{2} \frac{e^{(1-\tilde{a}) x}}{g(x)^{\frac{7}{5}-\tilde{a}}}+\sum_{i=1}^{2} \hat{p}_{i}{ }^{2} \frac{e^{\left(1-a_{i}\right) x}}{g(x)^{\frac{7}{5}-a_{i}}}
$$

The corresponding $0^{++}$mass spectrum is showed in Table 1 . 


\begin{tabular}{|c|c|c|}
\hline 0 & $\frac{\pi}{6}$ & $\frac{\pi}{12}$ \\
\hline 7.59 & 7.59 & 4.80 \\
\hline 10.40 & 10.40 & 7.81 \\
\hline 13.08 & 13.08 & 10.19 \\
\hline 15.71 & 15.71 & 12.41 \\
\hline 18.30 & 18.30 & 14.56 \\
\hline
\end{tabular}

Table 1: The table shows the values of $M_{0^{+}+}$mass, corresponding to dilatonic perturbations, for values $\beta=0, \beta=\frac{\pi}{6}$ and $\beta=\frac{\pi}{12}$ with $d=3$.

\subsection{Spectra from RR 1-form fluctuations.}

It is straightforward to verify that the perturbation defined by switching on only $f_{q+2}$, for any $q \neq D-2$ is consistent, and it is governed by the generalized Maxwell equations in (4.12). In particular, for $D=6$ we have just $a_{1}$ from the RR form sector. ${ }^{10}$.

From the Section 5, we analyze,

- Longitudinal polarizations: $0^{-+}$glueballs

By carrying out the same steps as in (4.19) we arrive to the Schrödinger form (4.21), with the potential,

$$
V(x)=\frac{1}{4 g(x)^{2}}\left(\frac{9}{25} e^{2 x}+\frac{2}{5}\left(3+2 a_{i}\right) e^{x}+a_{i}{ }^{2}\right)-\hat{M}^{2} \frac{e^{(1-\tilde{a}) x}}{g(x)^{\frac{7}{5}-\tilde{a}}}+\sum_{j \neq i} \hat{p}_{j}{ }^{2} \frac{e^{\left(1-a_{j}\right) x}}{g(x)^{\frac{7}{5}-a_{j}}}
$$

In Tables 2 we show the $0^{-+}$masses spectra for different polarizations.

\begin{tabular}{|c|c|c|}
\hline 0 & $\frac{\pi}{6}$ & $\frac{\pi}{12}$ \\
\hline 2.96 & 4.06 & 3.97 \\
\hline 5.55 & 6.69 & 6.67 \\
\hline 8.09 & 9.25 & 9.31 \\
\hline 10.61 & 11.78 & 11.94 \\
\hline 13.13 & 14.30 & 14.57 \\
\hline 15.64 & 16.82 & 17.18 \\
\hline
\end{tabular}

\begin{tabular}{|c|c|c|}
\hline 0 & $\frac{\pi}{6}$ & $\frac{\pi}{12}$ \\
\hline 4.06 & 2.96 & 3.79 \\
\hline 6.69 & 5.55 & 6.45 \\
\hline 9.25 & 8.09 & 9.08 \\
\hline 11.78 & 10.61 & 11.70 \\
\hline 14.31 & 13.12 & 14.32 \\
\hline 16.83 & 15.64 & 16.93 \\
\hline
\end{tabular}

Table 2: In the table on the left, we show the values of $M_{0^{-+}}$corresponding to longitudinal 1-form perturbation, polarized along direction characterized by $a_{1}$. The parameter takes values $\beta=0, \quad \beta=\frac{\pi}{6}$ and $\beta=\frac{\pi}{12}$. In the table on the right, we show these values for longitudinal polarization characterized by $a_{2}$. In both of them $d=3$.

- Transverse polarizations: $1^{++}$glueballs

\footnotetext{
${ }^{10}$ We would like to alert the reader that we work in a local basis, not in a coordinate one; therefore our tensor components differ from those in [19] by metric factors.
} 
The potential is,

$$
V(x)=\frac{1}{4 g(x)^{2}}\left(\frac{9}{25} e^{2 x}+\frac{2}{5}(3+2 \tilde{a}) e^{x}+\tilde{a}^{2}\right)-\hat{M}^{2} \frac{e^{(1-\tilde{a}) x}}{g(x)^{\frac{7}{5}-\tilde{a}}}+\sum_{j} \hat{p}_{j}{ }^{2} \frac{e^{\left(1-a_{j}\right) x}}{g(x)^{\frac{7}{5}-a_{j}}}
$$

We show in Table 3 the $1^{++}$mass spectra.

\begin{tabular}{|c|c|c|}
\hline 0 & $\frac{\pi}{6}$ & $\frac{\pi}{12}$ \\
\hline 2.96 & 2.96 & 3.10 \\
\hline 5.55 & 5.55 & 5.82 \\
\hline 8.09 & 8.09 & 8.47 \\
\hline 10.61 & 10.61 & 11.10 \\
\hline 13.12 & 13.12 & 13.72 \\
\hline
\end{tabular}

Table 3: The table shows the values of $M_{1^{++}}$, of transverse 1-form perturbation corresponding $\beta=0, \beta=\frac{\pi}{6}$ and $\beta=\frac{\pi}{12}$ for $d=3$.

\subsection{Glueball spectra from metric perturbations}

From the proposed metric ansätz in the section 5 we analyze, in the $d=3$, the following cases:

- Transverse polarizations: $2^{++}$glueballs

The potential,

$$
V(x)=\frac{1}{4}-\frac{1}{4 g(x)^{2}}-\hat{M}^{2} \frac{e^{(1-\tilde{a}) x}}{g(x)^{\frac{7}{5}-\tilde{a}}}
$$

We notice that there is no degeneration with the $0^{++}$spectrum as it happens in the critical case, a fact noted in [19] and that is valid for all the solutions of our family.

The corresponding $2^{++}$spectra is shown in Table 4 .

\begin{tabular}{|c|c|c|}
\hline 0 & $\frac{\pi}{6}$ & $\frac{\pi}{12}$ \\
\hline 4.06 & 4.06 & 4.28 \\
\hline 6.69 & 6.69 & 7.00 \\
\hline 9.25 & 9.25 & 9.66 \\
\hline 11.79 & 11.79 & 12.31 \\
\hline 14.31 & 14.3 & 14.94 \\
\hline
\end{tabular}

Table 4: In the table above, we show the values of $M_{2^{++}}$, corresponding to transverse metric perturbation, with $\beta=0, \beta=\frac{\pi}{6}$ and $\beta=\frac{\pi}{12}$. All of them were calculated with $d=3$. 
- Longitudinal polarizations: $1^{-+}$glueballs

The potential is,

$$
V(x)=\frac{1}{4}-\frac{1-\left(a_{i}-\tilde{a}\right)^{2}}{4 g(x)^{2}}-\hat{M}^{2} \frac{e^{(1-\tilde{a}) x}}{g(x)^{\frac{7}{5}-\tilde{a}}}
$$

In Tables 5 we show the $1^{-+}$masses spectra for different polarizations.

\begin{tabular}{|c|c|c|}
\hline 0 & $\frac{\pi}{6}$ & $\frac{\pi}{12}$ \\
\hline 4.06 & 5.00 & 5.06 \\
\hline 6.69 & 7.73 & 7.88 \\
\hline 9.25 & 10.34 & 10.59 \\
\hline 11.79 & 12.91 & 13.25 \\
\hline 14.31 & 15.45 & 15.90 \\
\hline 16.83 & 17.99 & 18.54 \\
\hline
\end{tabular}

\begin{tabular}{|c|c|c|}
\hline 0 & $\frac{\pi}{6}$ & $\frac{\pi}{12}$ \\
\hline 5.00 & 4.06 & 4.92 \\
\hline 7.73 & 6.69 & 7.70 \\
\hline 10.34 & 9.25 & 10.39 \\
\hline 12.91 & 11.79 & 13.051 \\
\hline 15.45 & 14.31 & 15.69 \\
\hline 17.99 & 16.83 & 18.33 \\
\hline
\end{tabular}

Table 5: In the table on the left, we show the values of $M_{1^{-+}}$, corresponding to longitudinal metric polarization along the direction characterized by $a_{1}$, for parameter values $\beta=0, \beta=\frac{\pi}{6}$ and $\beta=\frac{\pi}{12}$. In the table on the right, we show these values for polarization characterized by $a_{2}$. In both of them $d=3$.

- Scalars: $0^{++}$glueballs

The system $(5.18)$ is such that the element $(5.19)$ of the potential reduce to:

$$
\begin{aligned}
v(x) & =\frac{1}{4}-\frac{1}{4 g(x)^{2}}-\hat{M}^{2} \frac{e^{(1-\tilde{a}) x}}{g(x)^{1-\tilde{a}+\frac{2}{5}}} \\
m(x) & =\frac{2}{g(x)^{2}}\left(\frac{\tilde{a}}{2}(1-\tilde{a})+\frac{3 \tilde{a}-1}{5} e^{x}-\frac{2}{25} e^{2 x}\right) \\
m_{i}^{(1)}(x) & =\frac{1}{g(x)^{2}}\left(\frac{\tilde{a}}{2}\left(1+a_{i}-2 \tilde{a}\right)+\frac{2 \tilde{a}+a_{i}-1}{5} e^{x}-\frac{2}{25} e^{2 x}\right) \\
m_{i}^{(2)}(x) & =\frac{2}{g(x)^{2}}\left(\frac{a_{i}}{2}(1-\tilde{a})+\frac{4 a_{i}-\tilde{a}-1}{5} e^{x}-\frac{2}{25} e^{2 x}\right) \\
\mathbf{m}_{i j}(x) & =\frac{1}{g(x)^{2}}\left(\frac{a_{i}}{2}\left(1+a_{j}-2 \tilde{a}\right)+\frac{4 a_{i}+a_{j}-2 \tilde{a}-1}{5} e^{x}-\frac{2}{25} e^{2 x}\right)
\end{aligned}
$$

In Table 6, we show the $0^{++}$spectra corresponding to metric pertubations.

\section{Glueball spectra of $4 D$ Yang-Mills theories.}

We consider non critical IIA superstrings in $D=8$, in the background (2.2) of black $D 6$ branes. We will take $d=4$ equivalent directions, and $D-d-1=3$ as compact and non equivalent. The constraint equations (2.3) are,

$$
4 \tilde{a}+a_{1}+a_{2}+a_{3}=1 \quad, \quad 4 \tilde{a}^{2}+a_{1}^{2}+a_{2}^{2}+a_{3}^{2}=1
$$




\begin{tabular}{|c|c|c|}
\hline 0 & $\frac{\pi}{6}$ & $\frac{\pi}{12}$ \\
\hline 3.97 & 3.97 & 3.22 \\
\hline 6.67 & 6.67 & 6.36 \\
\hline 9.26 & 9.26 & 9.25 \\
\hline 11.81 & 11.81 & 12.04 \\
\hline 14.45 & 14.45 & 14.78 \\
\hline
\end{tabular}

Table 6: The table shows the values of $M_{0^{++}}$, corresponding to scalar metric perturbation for $\beta=0, \beta=\frac{\pi}{6}$ and $\beta=\frac{\pi}{12}$, and $d=3$.

They are explicitly solved by,

$$
\begin{aligned}
& a_{1}=\frac{1}{7}(\sqrt{21} x+\sqrt{15} z+1) \\
& a_{2}=\frac{1}{7}(-\sqrt{21} x+\sqrt{15} z+1) \\
& a_{3}=\frac{1}{7}\left(-2 \sqrt{\frac{42}{5}} y-\sqrt{\frac{12}{5}} z+1\right) \\
& 2 \tilde{a}=\frac{1}{7}\left(\sqrt{\frac{42}{5}} y-2 \sqrt{\frac{12}{5}} z+2\right)
\end{aligned}
$$

where

$$
x^{2}+y^{2}+z^{2}=1
$$

The parameter space results a two dimensional sphere characterized for example by standard angular variables $\theta$ and $\phi$.

As we made in the precedent section for the three dimensional models, let us look at the decoupling limit in this four dimensional case. The eight-dimensional Newton constant is $2 \kappa_{8}{ }^{2} \sim l_{s}{ }^{6} g_{s}{ }^{2}$, and then the decoupling of the tower of open string states as well as the bulk-open and bulk-bulk interactions requires the low energy limit $l_{s} \rightarrow 0$. And the question is focalized again on what remains on the world-volume of the $D 6$-brane. The non-critical vacuum preserves $2^{\frac{D}{2}}=16$ supercharges, and the BPS $D 6$-branes merged on it will preserve eight of them, as it can be argued following similar reasoning as in Section 0 from the knowledge that the low energy limit of a $D 5$-brane in the cigar vacuum is minimal $\mathcal{N}=(0,1)$ super Yang Mills in six dimensions, i.e., it preserves $8=\frac{1}{2} 16$ supercharges [23]. So we could argue that our family is holographic in the to UV some (unknown) CFT in seven dimensions, the completion of the seven dimensional YM theory whose coupling constant at scale $\Lambda_{s} \equiv l_{s}{ }^{-1}$ is,

$$
g_{Y M_{7}}{ }^{2}=\frac{T_{s}^{2}}{T_{D 6}} \sim l_{s}^{3} g_{s}
$$

The t'Hooft coupling at such scale, and the dimensionless effective coupling constant at 
scale $E$ are,

$$
\lambda_{7}^{2} \equiv g_{Y M_{7}}^{2} N \sim l_{s}^{3} \quad ; \quad \lambda_{7}^{e f f}(E)^{2} \equiv \lambda_{7}^{2} E^{3} \sim\left(\frac{E}{\Lambda_{s}}\right)^{3}
$$

from where the validity of the perturbative description in the region $E<<\Lambda_{s}$ and the absence of the decoupling limit follow [37]. The t'Hooft coupling at the compactification scale $\Lambda_{c} \equiv\left(8 \pi^{3} R_{1} R_{2} R_{1}\right)^{-\frac{1}{3}}$ of the four-dimensional theory we are interested in is,

$$
\lambda_{4}^{2}=\frac{\lambda_{7}^{2}}{8 \pi^{3} R_{1} R_{2} R_{3}} \sim\left(\frac{\Lambda_{c}}{\Lambda_{s}}\right)^{3}
$$

To make contact with four dimensional pure YM we should take the scaling limit [6] [7],

$$
\Lambda_{c} e^{-\frac{1}{B \lambda_{4}\left(\Lambda_{c}\right)}} \underset{\Lambda_{s} \rightarrow \infty}{\longleftrightarrow} \Lambda_{Q C D} \sim u_{0} \quad \longleftrightarrow \quad \ln \frac{\Lambda_{c}}{u_{0}} \sim\left(\frac{\Lambda_{s}}{\Lambda_{c}}\right)^{\frac{3}{2}}
$$

where $B$ is the coefficient of the one-loop beta function defined by, $\beta\left(\lambda_{4}\right) \equiv \mu \partial_{\mu} \lambda_{4}(\mu)=$ $-B \lambda_{4}^{2}+\ldots\left(B=\frac{23}{48 \pi^{2}}\right.$ for $S U(N)$ pure YM $)$.

We will not consider the $A_{3}^{+}$perturbation.

\subsection{Spectrum from dilatonic fluctuations.}

The Schröedinger like equation to solve for zero energy corresponds to the potential,

$$
V(x)=\frac{1}{4}-\frac{1}{4 g(x)^{2}}+\frac{8}{7} \frac{e^{x}}{g(x)}-\hat{M}^{2} \frac{e^{(1-\tilde{a}) x}}{g(x)^{\frac{9}{7}-\tilde{a}}}+\sum_{i=1}^{3} \hat{p}_{i}{ }^{2} \frac{e^{\left(1-a_{i}\right) x}}{g(x)^{\frac{9}{7}-a_{i}}}
$$

The corresponding mass spectra is shown in Table 7 .

\begin{tabular}{|c|c|c|}
\hline$P 1$ & $P 2$ & $P 3$ \\
\hline 10.69 & 11.15 & 11.43 \\
\hline 13.80 & 14.96 & 15.17 \\
\hline 16.78 & 18.4 & 18.68 \\
\hline 19.68 & 21.85 & 22.08 \\
\hline 22.54 & 25.18 & 25.67 \\
\hline
\end{tabular}

Table 7: The table shows the values of $M_{0^{++}}$mass, corresponding to dilatonic perturbations, in the parameter space points $P 1=\left(\frac{\pi}{6}, \frac{7 \pi}{6}\right), P 2=\left(\frac{\pi}{2}, \frac{4 \pi}{3}\right)$ and $P 3=\left(\frac{\pi}{3}, \frac{9 \pi}{6}\right)$ of the $d=4$ case.

\subsection{Spectra from RR 1-form fluctuations.}

It is straightforward to verify that the perturbation defined by switching on only $f_{q+2}$, for any $q \neq p+1$ is consistent, giving the generalized Maxwell equations (4.12). In particular, for $D=6$ we have just $a_{1}$ from the RR form sector. ${ }^{11}$.

From the Section 5, we analyze,

\footnotetext{
${ }^{11}$ We would like to alert the reader that we work in a local basis, not in a coordinate one; therefore our tensor components differ from those in 19 by metric factors.
} 


\begin{tabular}{|c|c|c|}
\hline$P 1$ & $P 2$ & $P 3$ \\
\hline 4.84 & 5.36 & 4.58 \\
\hline 7.68 & 8.25 & 7.43 \\
\hline 10.46 & 11.06 & 10.20 \\
\hline 13.21 & 13.84 & 12.95 \\
\hline
\end{tabular}

\begin{tabular}{|c|c|c|}
\hline$P 1$ & $P 2$ & $P 3$ \\
\hline 4.44 & 4.925 & 5.26 \\
\hline 7.29 & 7.74 & 8.12 \\
\hline 10.04 & 10.47 & 10.89 \\
\hline 12.75 & 13.19 & 13.62 \\
\hline
\end{tabular}

\begin{tabular}{|c|c|c|}
\hline$P 1$ & $P 2$ & $P 3$ \\
\hline 4.96 & 4.96 & 5.24 \\
\hline 7.85 & 7.85 &, 8.17 \\
\hline 10.66 & 10.66 & 11.00 \\
\hline 13.45 & 13.45 &, 13.80 \\
\hline
\end{tabular}

Table 8: In the table on the left, we show the values of $M_{0^{-+}}$, corresponding to longitudinal 1form perturbation polarized along direction characterized by $a_{1}$. The parameters take values on the 2-dimensional parameter space associated with $d=4$, named $P 1, P 2$ and $P 3$. In the tables on the center and on the right, we show these values for longitudinal polarization $a_{2}$ and $a_{3}$, respectively.

- Longitudinal polarizations: $0^{-+}$glueballs

By carrying out the same steps as in (4.19) we arrive to the Schrödinger form (4.20), with the potential,

$$
V(x)=\frac{1}{4 g(x)^{2}}\left(\frac{25}{49} e^{2 x}+\frac{2}{7}\left(5+2 a_{i}\right) e^{x}+{a_{i}}^{2}\right)-\hat{M}^{2} \frac{e^{(1-\tilde{a}) x}}{g(x)^{\frac{9}{7}-\tilde{a}}}+\sum_{j \neq i} \hat{p}_{j}{ }^{2} \frac{e^{\left(1-a_{j}\right) x}}{g(x)^{\frac{9}{7}-a_{j}}}
$$

In Tables 8 we show the $0^{-+}$masses spectra for different polarizations.

- Transverse polarizations: $1^{++}$glueballs

The potential is,

$$
V(x)=\frac{1}{4 g(x)^{2}}\left(\frac{25}{49} e^{2 x}+\frac{2}{7}(5+2 \tilde{a}) e^{x}+\tilde{a}^{2}\right)-\hat{M}^{2} \frac{e^{(1-\tilde{a}) x}}{g(x)^{\frac{9}{7}-\tilde{a}}}+\sum_{j} \hat{p}_{j}{ }^{2} \frac{e^{\left(1-a_{j}\right) x}}{g(x)^{\frac{9}{7}-a_{j}}}
$$

The corresponding $1^{++}$spectrum is shown in Table 9 .

\begin{tabular}{|c|c|c|}
\hline$P 1$ & $P 2$ & $P 3$ \\
\hline 4.42 & 4.31 & 4.52 \\
\hline 7.31 & 7.14 & 7.44 \\
\hline 10.10 & 9.89 & 10.27 \\
\hline 12.86 & 1260 & 13.06 \\
\hline 15.61 & 15.30 & 15.83 \\
\hline
\end{tabular}

Table 9: The table shows $M_{1++}$ values, of transverse 1-form perturbation at the points $P 1, \quad P 2$ and $P 3$ of the parameter space corresponding to $d=4$.

\subsection{Glueball spectra from metric perturbations}

From the proposed metric ansätz in the section 5 we analyze, in the $d=4$, the following cases: 
- Transverse polarizations: $2^{++}$glueballs

The potential,

$$
V(x)=\frac{1}{4}-\frac{1}{4 g(x)^{2}}-\hat{M}^{2} \frac{e^{(1-\tilde{a}) x}}{g(x)^{\frac{9}{7}-\tilde{a}}}
$$

We notice that there is no degeneration with the $0^{++}$spectrum as it happens in the critical case, a fact noted in [19] and that is valid for all the solutions of our family. The corresponding $2^{++}$mass spectrum is shown in Table 10.

\begin{tabular}{|c|c|c|}
\hline$P 1$ & $P 2$ & $P 3$ \\
\hline 5.52 & 5.40 & 5.60 \\
\hline 8.45 & 8.29 & 8.55 \\
\hline 11.27 & 11.07 & 11.40 \\
\hline 14.05 & 13.80 & 14.21 \\
\hline 16.81 & 16.52 & 17.00 \\
\hline
\end{tabular}

Table 10: In the table above, we show the values of $M_{2^{++}}$, corresponding to transverse metric perturbation. The values correspond to $P 1, P 2$ and $P 3$. All of them were calculated with $d=4$.

- Longitudinal polarizations: $1^{-+}$glueballs

The potential is,

$$
V(x)=\frac{1}{4}-\frac{1-\left(a_{i}-\tilde{a}\right)^{2}}{4 g(x)^{2}}-\hat{M}^{2} \frac{e^{(1-\tilde{a}) x}}{g(x)^{\frac{9}{7}-\tilde{a}}}
$$

In Tables 11 we show the $1^{-+}$masses spectra for different polarizations.

\begin{tabular}{|c|c|c|}
\hline$P 1$ & $P 2$ & $P 3$ \\
\hline 5.97 & 6.39 & 5.79 \\
\hline 8.93 & 9.42 & 8.72 \\
\hline 11.76 & 12.30 & 11.54 \\
\hline 14.55 & 15.12 & 14.32 \\
\hline
\end{tabular}

\begin{tabular}{|c|c|c|}
\hline$P 1$ & $P 2$ & $P 3$ \\
\hline 5.54, & 5.96 & 6.26 \\
\hline 8.41 & 8.87 & 9.23 \\
\hline 11.19 & 11.66 & 12.06 \\
\hline 13.92 & 14.41 & 14.82 \\
\hline
\end{tabular}

\begin{tabular}{|c|c|c|}
\hline$P 1$ & $P 2$ & $P 3$ \\
\hline 6.14 & 6.14 & 6.36 \\
\hline 9.14 & 9.14 & 9.41 \\
\hline 12.00 & 12.00 & 12.31 \\
\hline 14.83 & 14.83 & 15.15 \\
\hline
\end{tabular}

Table 11: In the tables above we show the values $M_{1^{-+}}$, of the longitudinal metric perturbation, along the three different directions associated with $a_{1}, a_{2}$ and $a_{3}$. The values correspond to points of parameter space that we have called $P 1, P 2$, and $P 3$ for $d=4$.

- Scalars: $0^{++}$glueballs

The system (5.18) is such that the element (5.19) of the potential reduce to:

$$
v(x)=\frac{1}{4}-\frac{1}{4 g(x)^{2}}-\hat{M}^{2} \frac{e^{(1-\tilde{a}) x}}{g(x)^{1-\tilde{a}+\frac{2}{7}}}
$$




$$
\begin{aligned}
m(x) & =\frac{4}{g(x)^{2}}\left(\frac{\tilde{a}}{2}(1-\tilde{a})+\frac{5 \tilde{a}-1}{7} e^{x}-\frac{2}{49} e^{2 x}\right) \\
m_{i}^{(1)}(x) & =\frac{1}{g(x)^{2}}\left(\frac{\tilde{a}}{2}\left(1+a_{i}-2 \tilde{a}\right)+\frac{4 \tilde{a}+a_{i}-1}{7} e^{x}-\frac{2}{49} e^{2 x}\right) \\
m_{i}^{(2)}(x) & =\frac{4}{g(x)^{2}}\left(\frac{a_{i}}{2}(1-\tilde{a})+\frac{6 a_{i}-\tilde{a}-1}{7} e^{x}-\frac{2}{49} e^{2 x}\right) \\
\mathbf{m}_{i j}(x) & =\frac{1}{g(x)^{2}}\left(\frac{a_{i}}{2}\left(1+a_{j}-2 \tilde{a}\right)+\frac{6 a_{i}+a_{j}-2 \tilde{a}-1}{7} e^{x}-\frac{2}{49} e^{2 x}\right)
\end{aligned}
$$

The corresponding $0^{++}$spectrum is shown in Table 12 .

\begin{tabular}{|c|c|c|}
\hline$P 1$ & $P 2$ & $P 3$ \\
\hline 6.44 & 6.37 & 6.50 \\
\hline 9.12 & 9.00 & 9.20 \\
\hline 11.62 & 11.47 & 11.72 \\
\hline 14.06 & 13.87 & 14.18 \\
\hline
\end{tabular}

Table 12: The table shows values of $M_{0^{++}}$, corresponding to scalar metric perturbation for three different points of the parameter space $P 1, P 2$, and $P 3$ with $d=4$.

\section{Summary of results and discussion}

We believe that is worth to start with some general remarks. First, it is an open question if such a thing like a low energy effective field theory associated to a non critical superstring theory, commonly referred in the literature as non critical supergravity, can be well defined. If it were so, presumably a scalar field (what would be a tachyon in the critical case, although in the present framework is just a misnomer) should also be present [30]. The existence of non critical superstrings lead us to conjecture that a manifestly supersymmetric action, maybe with infinite terms could be constructed. We think however that, from the results existing in the literature as well as those presented in this paper, the truncated action (2.1) usually considered is physically sensible. A further support to this statement is the existence, showed in [35], of a highly non trivial solution localized both in the Minkowski and cigar spaces, that was identified as the fundamental non critical string. Second, we think that the gauge-invariant, first order perturbation approach developed here in Section t 1 is a very interesting and useful tool because it is free of gauge dependencies and mixings, once a background is given.

Now let us go to the analysis of the results obtained in Section 6 and 7 . We note that, for the case $d=3$ we have two contributions to longitudinal polarizations, one coming from the direction associated with $a_{1}$ and the other from the one associated with $a_{2}$. These contributions are not KK-modes, but they are a consequence of the polarization along the two non-equivalent compact directions. Because of that, we have twice the states $0^{-+}$ 
and $1^{-+}$than those found in [19]. In general these modes are split (Figure 2), but for a few particular values of the parameter $\beta$ that labels the solutions (see (6.3)), they appear degenerated. The slightly splitting in mass is a direct consequence of the constraint (2.3).

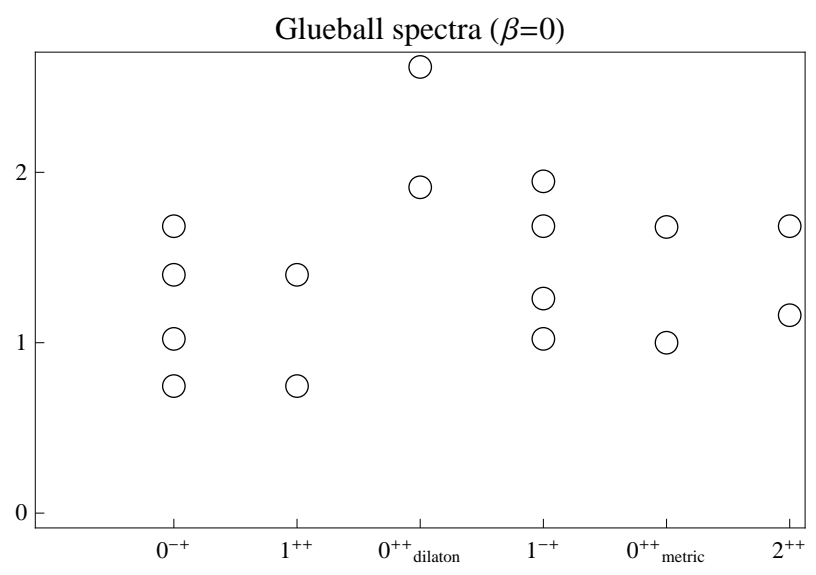

Figure 2: The plot shows the glueballs spectra normalized by the lightest mass of $0^{++}$of the metric perturbation in $d=3$, for $\beta=0$.

Even though we had hoped to reproduce the spectra obtained in [19] when the parameter $\beta$ becomes $\pi / 6$ (except for the splitting in longitudinal modes), this never happens. This is due to the difference between our lightest $0^{++}$mode related to metric perturbation and that obtained in [19]. We have found a better agreement than in [19] between the numerical and the WKB computations, and in virtue of this fact, we assume as a correct value for the lightest $0^{++}$mode of the scalar perturbation of the metric the one obtained here. Below, in the plot of Figure 3, we show the spectra obtained in [19] and the spectra obtained by us in the case $\beta=\frac{\pi}{6}$, each one normalized by their own lightest value of metric $0^{++}$. In the plot of Figure 4 , we show the same spectra, but now both are normalized by our lightest value of metric $0^{++}$. The agreement is perfect.

It is important to note that the expected qualitative aspect of the glueballs spectra is not that appearing in the previous plots for the particular values of $\beta$ that we have shown. In general it is widely assumed, and checked in some cases in Lattice QCD, that the lightest glueball corresponds to the operator $0^{++}$. Clearly, that is not the case for Figures 2 , 3 y 4. Nevertheless, because of the freedom in choosing the values of $\beta$, it is possible to tune the parameter to obtain a better agreement with the desired spectra.

Although it is not the aim of this paper to perform a systematic exploration of the confining sectors of the theories parametrized by $\beta$, it is possible to observe that some particular values of the parameter give a better agreement with the values obtained in Lattice QCD 41] (see Figure 66).

Finally, it is interesting to note that the same value of the parameter that provides the best agreement with Lattice QCD spectra (in the sense that the relative ratios between masses are more similar) is also the one for which the splitting in longitudinal polarized modes is smaller. We believe that a more accurate value of $\beta$ should be able to erase such splitting, leaving us with qualitatively and quantitatively more similar spectra to Lattice 


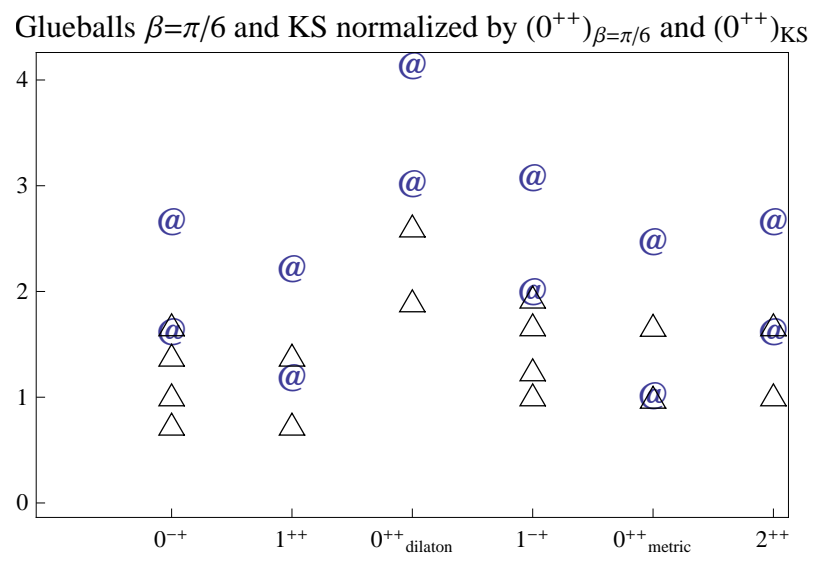

Figure 3: In this plot we compare the spectra obtained by Kuperstein and Sonnenschein [19](@) to our case for $\beta=\frac{\pi}{6}(\triangle)$, each one normalized by their corresponding lowest $0^{++}$. In principle, this two spectra should be the same except for the splitting in the longitudinal polarized modes $0^{-+}$and $1^{-+}$.

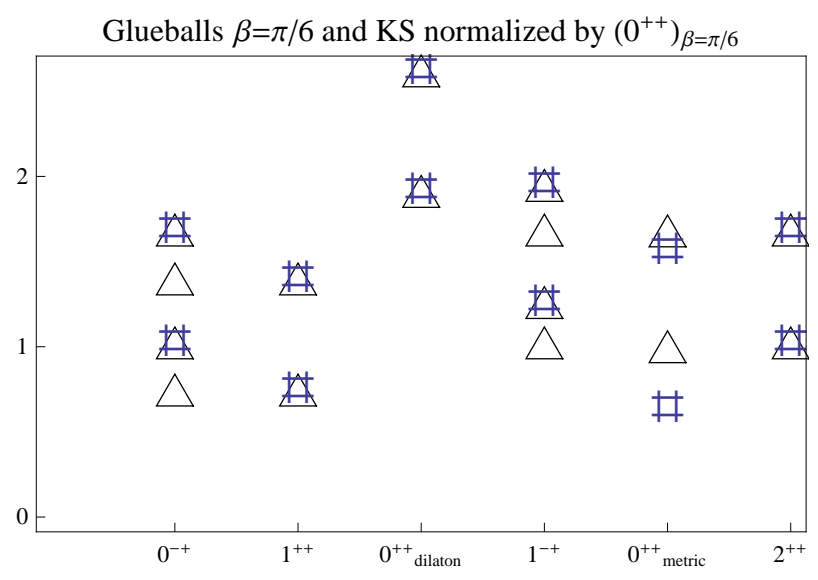

Figure 4: In this plot we compare the spectra obtained by Kuperstein and Sonnenschein [19]( $\sharp$ ) to our case for $\beta=\frac{\pi}{6}(\triangle)$, each one normalized by our lowest value of $0^{++}$. The agreement between the two spectra is perfect, except for the splitting in $0^{-+}$and $1^{-+}$.

QCD.

In the case of $d=4$ (Figure 7 ), our solutions have three non equivalent compact directions, and thus, three states with the same quantum numbers but different masses. As in the $d=3$ case, the splitting in the masses of longitudinal polarized modes appears as a consequence of the freedom in choosing the longitudinal direction along which to polarize the perturbation. In this case, we have two free parameters that characterize the solution (see (7.3)), and again this freedom enables us to obtain different spectra that we can compare with the results of Lattice QCD. Although it is very difficult to compute the entire spectrum for every point of the confining sector of the theory, that now is a 2-dimensional manifold, we think that a systematic exploration of the parameter space can be achieved with some numerical techniques, like Markov Chain Monte Carlo for example, 


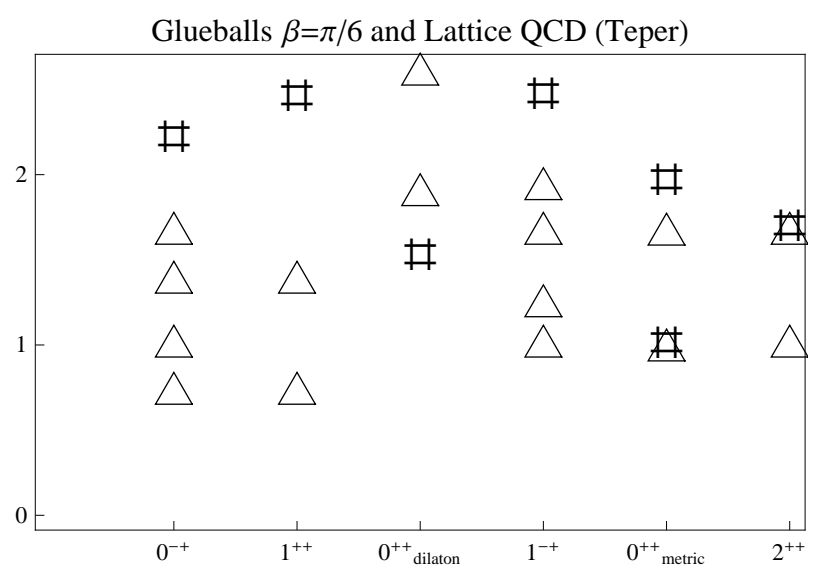

Figure 5: In this plot we compare our spectrum for $\beta=\pi / 6(\triangle)$ to the Lattice $Q C D(\sharp)$ spectrum obtained by Teper 41], for $d=3$.

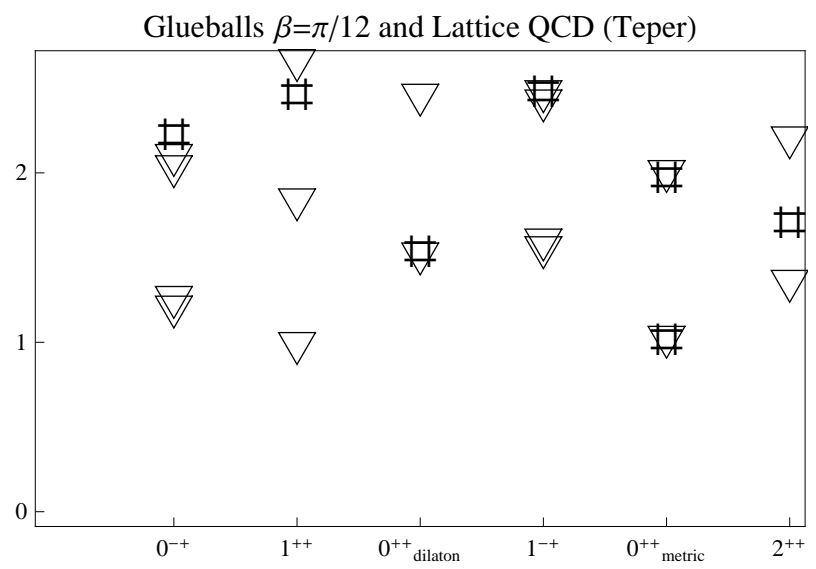

Figure 6: In this plot we compare our obtained spectrum for $\beta=\pi / 12(\nabla)$ with the Lattice $Q C D$ $(\sharp)$ obtained by Teper for $d=3(41])$.

and we hope that a better agreement with Lattice QCD can be obtained. In the present case, we selected in a random way the points in the confining sector of the parameter space of the theory (see Figure (8)).

We would like to remark as a very important fact that, although singular in the IR, all the solutions lead to a well-defined problem and spectra, without needing of extra boundary conditions at the singularity; results exist even when the solutions are singular in the IR limit. It is like if there were some mechanism at work, i.e. a barrier for string propagation in the backgrounds before the deep infrared region can be reached, as the Wilson loop computation in Section 3 seems to indicate. Furthermore, both families (2.2) and (B.1) yield exactly the same spectra as showed in Appendix (B), as one could guess from Tduality arguments ${ }^{12}$; however it results striking that while the string approximation for all the constant dilaton solutions in the family are under control in the large $\mathrm{N}$ limit, the T-dual family analysis seems to be restricted to the region $a_{\theta}<0$ due to the blow-up of

\footnotetext{
${ }^{12}$ We thank J. M. Maldacena for a discussion on this point.
} 


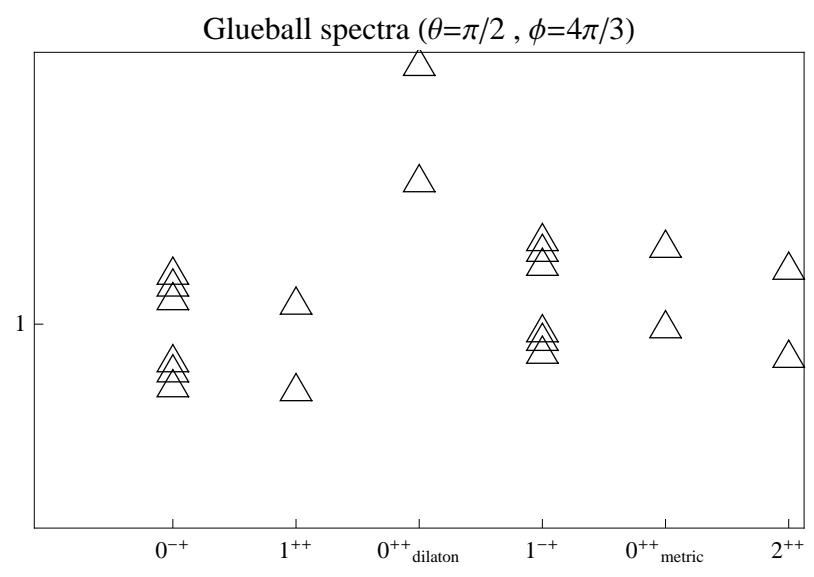

Figure 7: In this plot we show the glueballs spectra for $d=4$ normalized by the lowest masses of $0^{++}$for the point of the parameter space called $P 2$ that corresponds to $\theta=\frac{\pi}{2}$ and $\phi=\frac{4 \pi}{3}$.

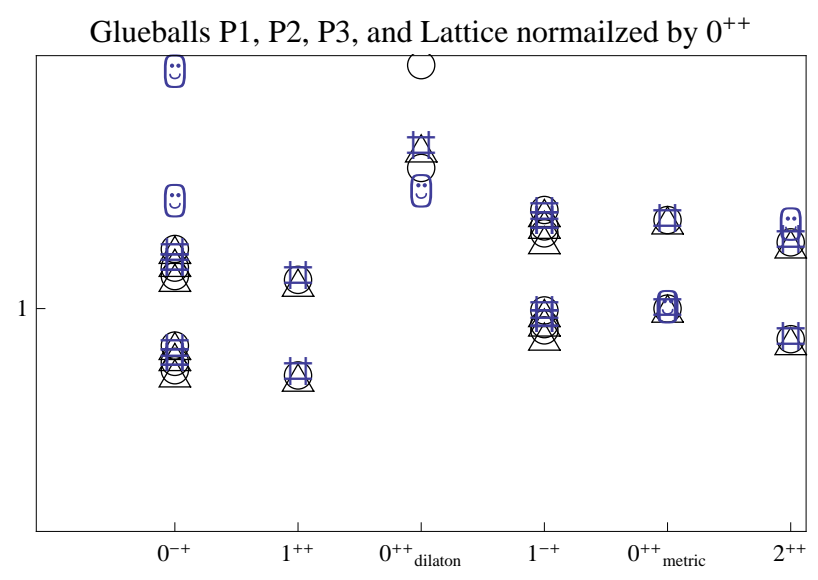

Figure 8: In the plot we show the glueballs spectra for $d=4$, computed for three different points of the parameter space $\mathrm{P} 1(\bigcirc), \mathrm{P} 2(\triangle), \mathrm{P} 3(\sharp)$ and we compare them with the Lattice $Q C D$ spectra $(\ddot{*})$ obtained by Morningstar and Peardon [42].

the effective string coupling $g_{s} \equiv e^{\Phi}$ in the infrared region $u \rightarrow u_{0}{ }^{+}$. We take this fact as a further sign of the effective irrelevance of the singularity. The repulsive character of IR singularities was noted in [43] (see [44] for a review).

It is possible that there exist exact solutions which approach our family in the UV but that are regular in the IR, maybe even at the level of the low energy effective action i.e. through a non trivial $\theta$-dependence, as much as it happens with the KlebanovTseytlin background [39] that presents a naked IR singularity that results regularized by the Klebanov-Strassler solution [40], but that modify in a mild way the spectra. Of course, a proof of this conjecture seems to be very far by now. ${ }^{13}$.

\footnotetext{
${ }^{13}$ An example of this that is not close to our set-up but similar in spirit is the Maldacena-Nunez solution [38], that regularize in the IR the back-reaction of $D 5$ - branes at the origin of the resolved conifold.
} 


\section{Acknowledgments}

We would like to thank Raúl Arias, Nicolás Grandi, Sameer Murthy, and Guillermo Silva for useful discussions, and the Abdus Salam ICTP of Trieste for kindly hospitality during part of this work.

\section{A. Some useful formulae.}

In this appendix we resume the conventions and formulae relevant in the computations carried out in the paper, Unless specified on the contrary, we work in a local basis with indices $A, B, C, \cdots=0,1, \ldots, D-1$.

Let us consider a metric of the form,

$$
G=\eta_{A B} \omega^{A} \omega^{B}=\eta_{a b} \omega^{a} \omega^{b}+\omega^{n 2} \quad, \quad a, b=0,1, \ldots, D-2
$$

where the vielbein $\omega^{A}$, dual vector fields $e_{A}, e_{A}\left(\omega^{B}\right)=\delta_{A}^{B}$, and volume element are,

$$
\begin{aligned}
\omega^{a} & =A_{a}(u) d x^{a} \quad, \quad e_{a}=A_{a}(u)^{-1} \partial_{a} \\
\omega^{n} & =C(u) d u \quad, \quad e_{n}=C(u)^{-1} \partial_{u} \\
\epsilon_{G} & =\omega^{0} \wedge \cdots \wedge \omega^{n}=d x^{0} \wedge \cdots \wedge d x^{D-2} \wedge d u E \quad, \quad E=\prod_{a} A_{a} C
\end{aligned}
$$

The pseudo-riemmanian connections, $\omega_{A B}=-\omega_{B A}: d \omega^{A}+\omega^{A}{ }_{B} \wedge \omega^{B}=0$ are,

$$
\begin{aligned}
\omega_{a b}=0 & , \quad \omega_{a n}=\sigma_{a} \omega_{a} \\
\sigma_{a} \equiv e_{n}\left(\ln A_{a}\right) & , \quad \sigma \equiv \sum_{a} \sigma_{a}=e_{n}\left(\ln \frac{E}{C}\right)
\end{aligned}
$$

The relevant covariant derivatives on a scalar field $\phi(x, u)$ are,

$$
\begin{aligned}
D_{A}(\phi) & =e_{A}(\phi) \quad, \quad \forall A \\
D_{a} D_{b}(\phi) & =e_{a} e_{b}(\phi)+\sigma_{a} e_{n}(\phi) \eta_{a b} \\
D_{a} D_{n}(\phi) & =e_{a} e_{n}(\phi)-\sigma_{a} e_{a}(\phi) \\
D_{n} D_{a}(\phi) & =e_{n} e_{a}(\phi)=D_{a} D_{n}(\phi) \\
D_{n}^{2}(\phi) & =e_{n}^{2}(\phi) \\
D^{2}(\phi) & =e^{a} e_{a}(\phi)+e_{n}^{2}(\phi)+\sigma e_{n}(\phi)=\sum_{a} \frac{\partial^{a} \partial_{a} \phi}{A_{a}^{2}}+\frac{1}{E} \partial_{u}\left(\frac{E}{C^{2}} \partial_{u} \phi\right)
\end{aligned}
$$

with $\sigma$ defined in (A.3).

The relevant covariant derivatives on a one-form $A=A_{A}(x, u) \omega^{A}$ are,

$$
\begin{aligned}
D_{b} A_{a} & =e_{b}\left(A_{a}\right)+\sigma_{b} A_{n} \eta_{a b} \\
D_{n} A_{a} & =e_{n}\left(A_{a}\right) \\
D_{a} A_{n} & =e_{a}\left(A_{n}\right)-\sigma_{a} A_{a} \\
D_{n} A_{n} & =e_{n}\left(A_{n}\right) \\
D_{c} D_{a} A_{b} & =e_{c} e_{a}\left(A_{b}\right)+\sigma_{c} e_{n}\left(A_{b}\right) \eta_{c a}-\sigma_{c} \sigma_{a} A_{a} \eta_{c b}+\sigma_{b}\left(e_{c}\left(A_{n}\right) \eta_{a b}+e_{a}\left(A_{n}\right) \eta_{c b}\right) \\
D_{n} D_{a} A_{b} & =e_{n} e_{a}\left(A_{b}\right)+e_{n}\left(\sigma_{a} A_{n}\right) \eta_{a b}
\end{aligned}
$$




$$
\begin{aligned}
& D_{a} D_{n} A_{b}=e_{a} e_{n}\left(A_{b}\right)-\sigma_{a} e_{a}\left(A_{b}\right)+\sigma_{b}\left(e_{n}\left(A_{n}\right)-\sigma_{b} A_{n}\right) \eta_{a b} \\
& D_{n} D_{n} A_{b}=e_{n}^{2}\left(A_{b}\right) \\
& D_{c} D_{a} A_{n}=e_{c} e_{a}\left(A_{n}\right)-\sigma_{a} e_{c}\left(A_{a}\right)-\sigma_{c} e_{a}\left(A_{c}\right)+\sigma_{a}\left(e_{n}\left(A_{n}\right)-\sigma_{a} A_{n}\right) \eta_{a c} \\
& D_{n} D_{a} A_{n}=e_{n} e_{a}\left(A_{n}\right)-e_{n}\left(\sigma_{a} A_{a}\right) \\
& D_{a} D_{n} A_{n}=e_{a} e_{n}\left(A_{n}\right)-\sigma_{a}\left(e_{a}\left(A_{n}\right)+e_{n}\left(A_{a}\right)-\sigma_{a} A_{a}\right) \\
& D_{n} D_{n} A_{n}=e_{n}^{2}\left(A_{n}\right)
\end{aligned}
$$

The relevant covariant derivatives on symmetric two-tensors $h=h_{A B}(x, u) \omega^{A} \omega^{B}$ are,

$$
\begin{aligned}
D_{c} h_{a b} & =e_{c}\left(h_{a b}\right)+\sigma_{c}\left(\eta_{a c} h_{b n}+\eta_{b c} h_{a n}\right) \\
D_{c} h_{a n} & =e_{c}\left(h_{a n}\right)+\sigma_{c}\left(\eta_{a c} h_{n n}-h_{a c}\right) \\
D_{c} h_{n n} & =e_{c}\left(h_{n n}\right)-2 \sigma_{c} h_{c n} \\
D_{n} h_{A B} & =e_{n}\left(h_{A B}\right) \quad, \quad \forall A, B \\
D_{d} D_{c} h_{a b} & =e_{d} e_{c}\left(h_{a b}\right)+\sigma_{c} \sigma_{d}\left(\left(\eta_{b c} \eta_{a d}+\eta_{a c} \eta_{b d}\right) h_{n n}-\eta_{a d} h_{b c}-\eta_{b d} h_{a c}\right) \\
& +\sigma_{c}\left(\eta_{c d} e_{n}\left(h_{a b}\right)+\eta_{a c} e_{d}\left(h_{b n}\right)+\eta_{b c} e_{d}\left(h_{a n}\right)+\sigma_{d}\left(\eta_{a d} e_{c}\left(h_{b n}\right)+\eta_{b d} e_{c}\left(h_{a n}\right)\right)\right. \\
D_{n} D_{c} h_{a b} & =e_{n} e_{c}\left(h_{a b}\right)+\eta_{a c} e_{n}\left(\sigma_{c} h_{b n}\right)+\eta_{b c} e_{n}\left(\sigma_{c} h_{a n}\right) \\
D_{c} D_{n} h_{a b} & =e_{c} e_{n}\left(h_{a b}\right)+\sigma_{c}\left(\eta_{a c} e_{n}\left(h_{b n}\right)+\eta_{b c} e_{n}\left(h_{a n}\right)-e_{c}\left(h_{a b}\right)\right)-\sigma_{c}^{2}\left(\eta_{a c} h_{b n}+\eta_{b c} h_{a n}\right) \\
D_{d} D_{c} h_{a n} & =e_{d} e_{c}\left(h_{a n}\right)-\sigma_{c} \sigma_{d}\left(\eta_{a c} h_{d n}+2 \eta_{a d} h_{c n}\right)+\sigma_{c}\left(\eta_{a c} e_{d}\left(h_{n n}\right)-e_{d}\left(h_{a c}\right)\right) \\
& +\sigma_{d}\left(\eta_{a d} e_{c}\left(h_{n n}\right)-e_{c}\left(h_{a d}\right)+\sigma_{d} \eta_{c d}\left(e_{n}\left(h_{a n}\right)-\sigma_{c} h_{a n}\right)\right. \\
D_{n} D_{c} h_{a n} & =e_{n} e_{c}\left(h_{a n}\right)+\eta_{a c} e_{n}\left(\sigma_{a} h_{n n}\right)-e_{n}\left(\sigma_{c} h_{a c}\right) \\
D_{c} D_{n} h_{a n} & =e_{c} e_{n}\left(h_{a n}\right)+\sigma_{c}\left(\eta_{a c} e_{n}\left(h_{n n}\right)-e_{n}\left(h_{a c}\right)-e_{c}\left(h_{a n}\right)\right)-\sigma_{c}^{2}\left(\eta_{a c} h_{n n}-h_{a c}\right) \\
D_{d} D_{c} h_{n n} & =e_{d} e_{c}\left(h_{n n}\right)-2 \sigma_{c} \sigma_{d}\left(\eta_{c d} h_{n n}-h_{c d}\right)+\sigma_{c} \eta_{c d} e_{n}\left(h_{n n}\right) \\
& -2\left(\sigma_{c} e_{d}\left(h_{c n}\right)+\sigma_{d} e_{c}\left(h_{d n}\right)\right) \\
D_{n} D_{c} h_{n n} & =e_{n} e_{c}\left(h_{n n}\right)-2 e_{n}\left(\sigma_{c} h_{c n}\right) \\
D_{c} D_{n} h_{n n} & =e_{c} e_{n}\left(h_{n n}\right)-\sigma_{c}\left(e_{c}\left(h_{n n}\right)+2\left(e_{n}\left(h_{c n}\right)-\sigma_{c} h_{c n}\right)\right) \\
D_{n} D_{n} h_{A B} & =e_{n}^{2}\left(h_{A B}\right) \quad, \quad \forall A, B
\end{aligned}
$$

The curvature tensor $\Re_{A B} \equiv d \omega_{A B}+\omega_{A C} \wedge \omega^{C}{ }_{B}$ is,

$$
\begin{aligned}
\Re_{a b} & =-\sigma_{a} \sigma_{b} \omega_{a} \wedge \omega_{b} \\
\Re_{a n} & =-\frac{1}{A_{a}} e_{n}^{2}\left(A_{a}\right) \omega_{a} \wedge \omega_{n}
\end{aligned}
$$

and the Ricci tensor and scalar are,

$$
\begin{aligned}
R_{a b} & =-D^{2}\left(\ln A_{a}\right) \eta_{a b} \\
R_{n n} & =-\sum_{a} \frac{1}{A_{a}} e_{n}{ }^{2}\left(A_{a}\right)=-\left(e_{n}(\sigma)+\sum_{a} \sigma_{a}{ }^{2}\right) \\
R & =-2 D^{2}\left(\ln \frac{E}{C}\right)+\sigma^{2}-\sum_{a} \sigma_{a}{ }^{2}
\end{aligned}
$$

\section{A.1 Computation of the tensor $A_{A B}$}

The following symmetric two-tensor,

$$
A_{A B}(h) \equiv D_{A} D_{B} h_{C}^{C}+D^{2} h_{A B}-D^{C} D_{A} h_{C B}-D^{C} D_{B} h_{A C}
$$


naturally arises in the metric perturbation theory. With the help of (A.6) we get the expressions,

$$
\begin{aligned}
A_{a b}(h) & =e^{A} e_{A}\left(h_{a b}\right)+e_{a} e_{b}\left(h_{C}^{C}\right)-e_{a} e_{n}\left(h_{b n}\right)-e_{b} e_{n}\left(h_{a n}\right)-e^{c} e_{a}\left(h_{b c}\right)-e^{c} e_{b}\left(h_{a c}\right) \\
& +\sigma e_{n}\left(h_{a b}\right)+\left(e_{n}\left(\sigma_{a}+\sigma_{b}\right)+\sigma\left(\sigma_{a}+\sigma_{b}\right)-\left(\sigma_{a}-\sigma_{b}\right)^{2}\right) h_{a b} \\
& +\left(2 \sigma_{a}-\sigma_{b}-\sigma\right) e_{a}\left(h_{b n}\right)+\left(2 \sigma_{b}-\sigma_{a}-\sigma\right) e_{b}\left(h_{a n}\right) \\
& +\eta_{a b}\left(-2\left(e_{n}\left(\sigma_{a}\right)+\sigma \sigma_{a}\right) h_{n n}+\sigma_{a}\left(e_{n}\left(h_{c}^{c}\right)-e_{n}\left(h_{n n}\right)-2 e^{c}\left(h_{c n}\right)\right)\right. \\
A_{a n}(h) & =e^{c} e_{c}\left(h_{a n}\right)-e^{c} e_{n}\left(h_{a c}\right)-e^{c} e_{a}\left(h_{c n}\right)+e_{n} e_{a}\left(h_{c}^{c}\right)+2\left(e_{n}\left(\sigma_{a}\right)+\sigma \sigma_{a}\right) h_{a n} \\
& -\left(\sigma-\sigma_{a}\right) e_{a}\left(h_{n n}\right)+\left(\sigma_{a}-\sigma_{c}\right) e^{c}\left(h_{a c}\right)+\sigma_{c} e_{a}\left(h_{c}^{c}\right) \\
A_{n n}(h) & =e^{c} e_{c}\left(h_{n n}\right)-\sigma e_{n}\left(h_{n n}\right)-2 e^{c} e_{n}\left(h_{c n}\right)-2 \sigma_{c} e^{c}\left(h_{c n}\right)+e_{n}{ }^{2}\left(h_{c}^{c}\right)+2 \sigma_{c} e_{n}\left(h_{c}^{c}\right)
\end{aligned}
$$

Under the gauge transformation in (4.9),

$$
\begin{aligned}
A_{a b}\left(\delta_{\epsilon} h\right) & =\left(e_{n}\left(\sigma_{a}\right)+\sigma \sigma_{a}+e_{n}\left(\sigma_{b}\right)+\sigma \sigma_{b}\right) \delta_{\epsilon} h_{a b}+2 e_{n}\left(e_{n}\left(\sigma_{a}\right)+\sigma \sigma_{a}\right) \epsilon_{n} \eta_{a b} \\
& +\left(e_{n}\left(\sigma_{b}\right)+\sigma \sigma_{b}-e_{n}\left(\sigma_{a}\right)-\sigma \sigma_{a}\right)\left(e_{a}\left(\epsilon_{b}\right)-e_{b}\left(\epsilon_{a}\right)\right) \\
A_{a n}\left(\delta_{\epsilon} h\right) & \left.=2\left(e_{n}\left(\sigma_{a}\right)+\sigma \sigma_{a}\right) \delta_{\epsilon} h_{a n}+2\left(\sum_{n}\left(\sigma_{c}\right)+\sigma_{c}^{2}\right)-e_{n}\left(\sigma_{a}\right)-\sigma \sigma_{a}\right) e_{a}\left(\epsilon_{n}\right) \\
A_{n n}\left(\delta_{\epsilon} h\right) & =2 \sum_{c}\left(e_{n}\left(\sigma_{c}\right)+\sigma_{c}^{2}\right) \delta_{\epsilon} h_{n n}+2 e_{n}\left(\sum_{c}\left(e_{n}\left(\sigma_{c}\right)+\sigma_{c}^{2}\right)\right) \epsilon_{n}
\end{aligned}
$$

\section{A.2 A short derivation of the solutions.}

Here we sketch the obtention of the family of solutions (2.2) considered in the paper. Let us consider an ansätz for the metric of the form (A.1), together with,

$$
\begin{aligned}
\Phi(u) & =\Phi=\text { constant } \\
F_{D} & =(-)^{D} Q_{D-2} \epsilon_{G} \Longleftrightarrow * F_{D}=(-)^{D-1} Q_{D-2}
\end{aligned}
$$

The strength field tensor in (A.12) leads to,

$$
\left(F_{D}\right)_{A B}^{2}=\left(F_{D}\right)^{2} \eta_{A B} \quad, \quad\left(F_{D}\right)^{2}=-\left(\frac{C e_{n}(E)}{E}\right)^{2}
$$

The equations of motion in string frame read (we consider D-branes, $b_{D-2}=0$ ),

$$
\begin{aligned}
R_{A B} & =\frac{1}{4} e^{2 \Phi}\left(F_{D}\right)^{2} \eta_{A B} \\
\Lambda^{2} & =-\frac{D}{4} e^{2 \Phi}\left(F_{D}\right)^{2} \\
d\left(* F_{D}\right) & =(-)^{D} Q_{D-2} * J_{D-1} \quad, \quad Q_{D-2} \equiv 2 \kappa_{D}^{2} \mu_{D-2}
\end{aligned}
$$

where $\kappa_{D}^{2}=8 \pi G_{D}$ is the gravitational coupling and $\mu_{D-2}$ the $\mathrm{D}(D-2)$-brane tension. The last two equations are solved by,

$$
\frac{C e_{n}(E)}{E}=-Q_{D-2} \quad, \quad e^{2 \Phi}=\frac{4}{D} \frac{\Lambda^{2}}{Q_{D-2}{ }^{2}}
$$


while that the metric equations reduce to,

$$
\begin{aligned}
D^{2}\left(\ln A_{a}\right) \equiv e_{n}\left(\sigma_{a}\right)+\sigma \sigma_{a} & =\frac{\Lambda^{2}}{D} \quad, \quad \forall a \\
\sum_{a}\left(e_{n}\left(\sigma_{a}\right)+\sigma_{a}{ }^{2}\right) & =\frac{\Lambda^{2}}{D}
\end{aligned}
$$

By following steps similar to those in reference [21], the general solution to (A.16) can be written,

$$
A_{a}(u)=l_{0} u f(u)^{\frac{a_{a}}{2}} \quad ; \quad C(u)=l_{0} u^{-1} f(u)^{-\frac{1}{2}}
$$

with $f(u)$ as in (2.2), and the constraints on the exponents given in (2.3).

\section{B. The fluctuations in the T-dual solutions.}

In this appendix we present the analysis of the perturbations around the backgrounds Tdual to those considered in the main of the paper. It results more involved due to the presence of a non constant dilaton, however yields the same spectra.

\section{B.1 The family of solutions.}

The family of solutions (in string frame) obtained from (2.2) by performing a T-duality transformation in, i.e. $x^{D-2}-$ coordinate reads,

$$
\begin{aligned}
l_{0}^{-2} G & =u^{2} f(u)^{a_{\mu}} \eta_{\mu \nu} d x^{\mu} d x^{\nu}+\frac{d u^{2}}{u^{2} f(u)}+u_{1}^{2} \frac{d \theta^{2}}{u^{2} f(u)^{a_{\theta}}} \quad, \quad \Lambda^{2} l_{0}^{2}=D(D-1) \\
e^{\Phi} & =\frac{4 \pi \sqrt{D-1}}{\left|Q_{D-3}\right|} \frac{u_{1}}{u} f(u)^{-\frac{a_{\theta}}{2}} \\
F_{D-1} & =\frac{Q_{D-3} l_{0} D-2}{2 \pi u_{1}} u^{D-2} d u \wedge d x^{0} \wedge \cdots \wedge d x^{D-3} \leftrightarrow * F_{D-1}=(-)^{D-1} Q_{D-3} \frac{d \theta}{2 \pi}
\end{aligned}
$$

where $\mu, \nu=0,1, \ldots, D-3$, the coordinate $\theta$ is $2 \pi$-periodic, and the identifications

$$
f(u)=1-\left(\frac{u_{0}}{u}\right)^{D-1}
$$

The scales $u_{0}$ and $u_{1}$ are arbitrary, and Furthermore, the following constraints on the exponents must hold,

$$
\sum_{\mu} a_{\mu}+a_{\theta}=1 \quad, \quad \sum_{\mu} a_{\mu}^{2}+a_{\theta}^{2}=1
$$

These solutions could be interpreted as the near horizon of $\mathrm{D}(\mathrm{D}-3)$-branes in a linear dilaton background.

Some useful relations are,

$$
e_{n}\left(\sigma_{\mu}\right)+\sigma \sigma_{\mu}=\frac{\Lambda^{2}}{D-2} e^{\frac{4}{D-2} \Phi}
$$




$$
\begin{aligned}
e_{n}\left(\sigma_{\theta}\right)+\sigma \sigma_{\theta} & =-\frac{D-4}{D(D-2)} \Lambda^{2} e^{\frac{4}{D-2} \Phi} \\
\sum_{a}\left(e_{n}\left(\sigma_{a}\right)+\sigma_{a}^{2}\right) & =\frac{\Lambda^{2}}{D-2} e^{\frac{4}{D-2} \Phi}-\frac{4(D-2)}{(D-4)^{2}} \sigma_{\theta}^{2} \\
\sigma^{2}-\sum_{a} \sigma_{a}^{2} & =\frac{D-2}{D} \Lambda^{2} e^{\frac{4}{D-2} \Phi}+\frac{4(D-2)}{(D-4)^{2}} \sigma_{\theta}^{2} \\
e_{n}^{2}(\Phi)+\sigma e_{n}(\Phi) & =-\frac{\Lambda^{2}}{D} e^{\frac{4}{D-2} \Phi} \\
e_{n}(\Phi) & =\frac{D-2}{D-4} \sigma_{\theta}
\end{aligned}
$$

\section{B.2 The equations for the fluctuations in e-frame}

As remarked in Section 4, since the system for the fluctuations is linear, it should be possible to write it in a manifest gauge invariant way under (4.9). For the family (B.1) that will concern us here we can do it as follows. First, we restrict ourselves to fluctuations such that,

$$
f_{D-1} \equiv \chi F_{D-1} \quad, \quad{ }^{\epsilon} \chi=\chi+D_{\tilde{a}} \epsilon^{\tilde{a}}+\frac{D}{D-4} \sigma_{\theta} \epsilon_{n}
$$

Consistency with the Bianchi identity imposes $\theta$-independence of $\chi$, and therefore on all the fluctuations; moreover the gauge transformation of $\chi$ follows from (4.9). In second term, following (4.28) we introduce the fields $\left(g, g_{a}, I_{a b}, I_{\xi}, I_{\chi}\right)$ by,

$$
\begin{aligned}
h_{n n} & \equiv 2 e_{n}(g) & , & \delta_{\epsilon} g=\epsilon_{n} \\
h_{a n} & \equiv A_{a} e_{n}\left(\frac{g_{a}}{A_{a}}\right)+e_{a}(g) & , & \delta_{\epsilon} g_{a}=\epsilon_{a} \\
h_{a b} & \equiv I_{a b}+e_{a}\left(g_{b}\right)+e_{b}\left(g_{a}\right)+2 \eta_{a b} \sigma_{a} g & , & \delta_{\epsilon} I_{a b}=0 \\
\xi & \equiv I_{\xi}+\frac{D-2}{D-4} \sigma_{\theta} g & & \delta_{\epsilon} I_{\xi}=0 \\
\chi & \equiv I_{\chi}+e^{\mu}\left(g_{\mu}\right)+e_{n}(g)+\left(\sigma+\frac{4}{D-4} \sigma_{\theta}\right) g & , & \delta_{\epsilon} I_{\chi}=0
\end{aligned}
$$

where again the gauge transformations follows from (4.9). In terms of these variables (A.10) is written as,

$$
\begin{aligned}
A_{a b}(h) & =A_{a b}^{(i)}(I)+2\left(e_{n}\left(\sigma_{b}\right)+\sigma \sigma_{b}\right) e_{a}\left(g_{b}\right)+2\left(e_{n}\left(\sigma_{a}\right)+\sigma \sigma_{a}\right) e_{b}\left(g_{a}\right) \\
& +2 \eta_{a b}\left(e_{n}\left(e_{n}\left(\sigma_{a}\right)+\sigma \sigma_{a}\right)+2 \sigma_{a}\left(e_{n}\left(\sigma_{a}\right)+\sigma \sigma_{a}\right)\right) g \\
A_{a b}^{(i)}(I) & \equiv e^{A} e_{A}\left(I_{a b}\right)+e_{a} e_{b}\left(I_{c}^{c}\right)-e_{a} e^{c}\left(I_{b c}\right)-e_{b} e^{c}\left(I_{a c}\right)+\sigma e_{n}\left(I_{a b}\right) \\
& +\left(e_{n}\left(\sigma_{a}+\sigma_{b}\right)+\sigma\left(\sigma_{a}+\sigma_{b}\right)-\left(\sigma_{a}-\sigma_{b}\right)^{2}\right) I_{a b}+\eta_{a b} \sigma_{a} e_{n}\left(I_{c}^{c}\right) \\
A_{a n}(h) & =A_{a n}^{(i)}(I)+2\left(e_{n}\left(\sigma_{a}\right)+\sigma \sigma_{a}\right)\left(e_{n}\left(g_{a}\right)-\sigma_{a} g_{a}\right)+2 \sum_{c}\left(e_{n}\left(\sigma_{c}\right)+\sigma_{c}^{2}\right) e_{a}(g) \\
A_{a n}^{(i)}(I) & \equiv-e^{c} e_{n}\left(I_{a c}\right)+e_{a} e_{n}\left(I_{c}^{c}\right)+\left(\sigma_{a}-\sigma_{c}\right)\left(e^{c}\left(I_{a c}\right)-e_{a}\left(I_{c}^{c}\right)\right) \\
A_{n n}(h) & =A_{n n}^{(i)}(I)+4 \sum_{c}\left(e_{n}\left(\sigma_{c}\right)+\sigma_{c}^{2}\right) e_{n}(g)+2 e_{n}\left(\sum_{c}\left(e_{n}\left(\sigma_{c}\right)+\sigma_{c}^{2}\right)\right) g \\
A_{n n}^{(i)}(I) & \equiv e_{n}{ }^{2}\left(I_{c}^{c}\right)+2 \sigma_{c} e_{n}\left(I_{c}^{c}\right)
\end{aligned}
$$


where the $A_{A B}^{(i)}(I)^{\prime}$ 's are manifest gauge invariant; we remark that these expressions are valid for any metric of the form in (A.1). After a lengthy but straightforward computation equations (4.5), (4.6), (4.7) in our backgrounds (B.1) result,

h-equations

$$
\begin{aligned}
0 & =e^{A} e_{A}\left(I_{\mu \nu}\right)+e_{\mu} e_{\nu}\left(I_{c}^{c}\right)-e_{\mu} e^{c}\left(I_{\nu c}\right)-e_{\nu} e^{c}\left(I_{\mu c}\right)+\sigma e_{n}\left(I_{\mu \nu}\right) \\
& +\eta_{\mu \nu} \sigma_{\mu} e_{n}\left(I_{c}^{c}\right)-\left(\sigma_{\mu}-\sigma_{\nu}\right)^{2} I_{\mu \nu}-\frac{8 \Lambda^{2}}{(D-2)^{2}} \eta_{\mu \nu} e^{\frac{4}{D-2} \Phi} I_{\xi} \\
0 & =-e^{c} e_{n}\left(I_{\mu c}\right)+e_{\mu} e_{n}\left(I_{c}^{c}\right)+\left(\sigma_{\mu}-\sigma_{c}\right)\left(e^{c}\left(I_{\mu c}\right)-e_{\mu}\left(I_{c}^{c}\right)\right)+\frac{8}{D-4} \sigma_{\theta} e_{\mu}\left(I_{\xi}\right) \\
0 & =e_{n}^{2}\left(I_{c}^{c}\right)+2 \sigma_{c} e_{n}\left(I_{c}^{c}\right)+\frac{16}{D-4} \sigma_{\theta} e_{n}\left(I_{\xi}\right)-\frac{8 \Lambda^{2}}{(D-2)^{2}} e^{\frac{4}{D-2} \Phi} I_{\xi} \\
0 & =e^{A} e_{A}\left(I_{\theta \theta}\right)+\sigma e_{n}\left(I_{\theta \theta}\right)+\sigma_{\theta} e_{n}\left(I_{c}^{c}\right)+\frac{8 \Lambda^{2}}{D} e^{\frac{4}{D-2} \Phi}\left(I_{\chi}-\frac{1}{2} I_{\mu}^{\mu}-\frac{D^{2}-3 D+4}{(D-2)^{2}} I_{\xi}\right) \\
0 & =e^{A} e_{A}\left(I_{\mu \theta}\right)+\sigma e_{n}\left(I_{\mu \theta}\right)-e_{\mu} e^{c}\left(I_{c \theta}\right)+\left(\frac{2 \Lambda^{2}}{D} e^{\frac{4}{D-2} \Phi}-\left(\sigma_{\mu}-\sigma_{\theta}\right)^{2}\right) I_{\mu \theta} \\
0 & =e^{c}\left(e_{n}\left(I_{c \theta}\right)+\left(\sigma_{c}-\sigma_{\theta}\right) I_{c \theta}\right)
\end{aligned}
$$

$\underline{\xi \text {-equation }}$

$$
\begin{aligned}
0 & =e^{A} e_{A}\left(I_{\xi}\right)+\sigma e_{n}\left(I_{\xi}\right)+\frac{D-2}{2(D-4)} \sigma_{\theta} e_{n}\left(I_{c}^{c}\right) \\
& +\frac{D-2}{D} \Lambda^{2} e^{\frac{4}{D-2} \Phi}\left(-I_{\chi}+\frac{1}{2} I_{\mu}^{\mu}+\frac{D^{2}-2 D+4}{(D-2)^{2}} I_{\xi}\right)
\end{aligned}
$$

$\underline{a_{q+1} \text {-equation }, \quad q \neq D-3}$

$$
0=-e^{-\alpha_{q} \Phi} D^{B}\left(e^{\alpha_{q} \Phi}\left(f_{q+2}\right)_{A_{1} \ldots A_{q+1} B}\right)
$$

$\underline{a_{D-2} \text {-equation }}$

From (4.7) we get an equation with $(D-2)$ antisymmetric indices that leads to the following equations,

$$
\begin{aligned}
& 0=e_{\mu}\left(I_{\nu \theta}\right)-e_{\nu}\left(I_{\mu \theta}\right) \quad, \quad \forall \mu, \nu \\
& 0=e_{n}\left(\frac{A_{\mu}}{A_{\theta}} I_{\mu \theta}\right) \quad, \quad \forall \mu \\
& 0=e_{\tilde{a}}\left(2 I_{\xi}-I_{\chi}+\frac{1}{2}\left(I_{\mu}^{\mu}-I_{\theta \theta}\right)\right) \quad, \quad \forall \tilde{a}
\end{aligned}
$$

with solution,

$$
\begin{aligned}
& I_{\mu \theta}=0 \quad, \quad \forall \mu \\
& I_{\chi}=2 I_{\xi}+\frac{1}{2}\left(I_{\mu}^{\mu}-I_{\theta \theta}\right)
\end{aligned}
$$


The fluctuations $a_{q+1}$ with $q \neq D-3$ are gauge invariant and decoupled from the rest of the fluctuations; we focus on these last ones. By using the relations (B.11) in (B.8), (B.9) we get the following system,

$$
\begin{aligned}
0 & =e_{n}^{2}\left(I_{\mu \nu}\right)+\sigma e_{n}\left(I_{\mu \nu}\right)+e^{\rho} e_{\rho}\left(I_{\mu \nu}\right)+e_{\mu} e_{\nu}\left(I_{c}^{c}\right)-e_{\mu} e^{\rho}\left(I_{\nu \rho}\right)-e_{\nu} e^{\rho}\left(I_{\mu \rho}\right)-\left(\sigma_{\mu}-\sigma_{\nu}\right)^{2} I_{\mu \nu} \\
& +\eta_{\mu \nu} \sigma_{\mu} e_{n}\left(I_{c}^{c}\right)-\frac{8 \Lambda^{2}}{(D-2)^{2}} \eta_{\mu \nu} e^{\frac{4}{D-2} \Phi} I_{\xi} \\
0 & =e_{n}^{2}\left(I_{\theta \theta}\right)+\sigma e_{n}\left(I_{\theta \theta}\right)+e^{\rho} e_{\rho}\left(I_{\theta \theta}\right)+\sigma_{\theta} e_{n}\left(I_{c}^{c}\right) \\
& +\frac{8 \Lambda^{2}}{D} e^{\frac{4}{D-2} \Phi}\left(\frac{(D-1)(D-4)}{(D-2)^{2}} I_{\xi}-\frac{1}{2} I_{\theta \theta}\right) \\
0 & =-e^{\rho} e_{n}\left(I_{\mu \rho}\right)+e_{\mu} e_{n}\left(I_{c}^{c}\right)+\left(\sigma_{\mu}-\sigma_{\rho}\right) e^{\rho}\left(I_{\mu \rho}\right)+\left(\sigma_{c}-\sigma_{\mu}\right) e_{\mu}\left(I_{c}^{c}\right)+\frac{8}{D-4} \sigma_{\theta} e_{\mu}\left(I_{\xi}\right) \\
0 & =e_{n}^{2}\left(I_{c}^{c}\right)+2 \sigma_{c} e_{n}\left(I_{c}^{c}\right)+\frac{16}{D-4} \sigma_{\theta} e_{n}\left(I_{\xi}\right)-\frac{8 \Lambda^{2}}{(D-2)^{2}} e^{\frac{4}{D-2} \Phi} I_{\xi} \\
0 & =D^{2}\left(I_{\xi}\right)+\frac{D-2}{2(D-4)} \sigma_{\theta} e_{n}\left(I_{c}^{c}\right)-\frac{(D-2) \Lambda^{2}}{D} e^{\frac{4}{D-2} \Phi}\left(\frac{D^{2}-6 D+4}{(D-2)^{2}} I_{\xi}-\frac{1}{2} I_{\theta \theta}\right)
\end{aligned}
$$

Now we introduce the modes in momenta,

$$
I_{\mu \nu}=\chi_{\mu \nu}(u) e^{i p \cdot x} \quad ; \quad I_{\theta \theta}=\chi_{\theta}(u) e^{i p \cdot x} \quad ; \quad I_{\xi}=\chi_{\xi}(u) e^{i p \cdot x}
$$

where $p \cdot x \equiv p_{\rho} x^{\rho}$. With the definition $p_{\mu} \equiv A_{\mu} \tilde{p}_{\mu}$, we get the system in the form,

$$
\begin{aligned}
0 & =e_{n}^{2}\left(\chi_{\mu \nu}\right)+\sigma e_{n}\left(\chi_{\mu \nu}\right)-\left(\tilde{p}^{\rho} \tilde{p}_{\rho}+\left(\sigma_{\mu}-\sigma_{\nu}\right)^{2}\right) \chi_{\mu \nu}+\tilde{p}_{\mu} \tilde{p}^{\rho} \chi_{\nu \rho}+\tilde{p}_{\nu} \tilde{p}^{\rho} \chi_{\mu \rho} \\
& -\tilde{p}_{\mu} \tilde{p}_{\nu}\left(\chi_{\rho}^{\rho}+\chi_{\theta}\right)+\eta_{\mu \nu} \sigma_{\mu} e_{n}\left(\chi_{\rho}^{\rho}+\chi_{\theta}\right)-\frac{8 \Lambda^{2}}{(D-2)^{2}} \eta_{\mu \nu} e^{\frac{4}{D-2} \Phi} \chi_{\xi} \\
0 & =e_{n}^{2}\left(\chi_{\theta}\right)+\sigma e_{n}\left(\chi_{\theta}\right)-\left(\tilde{p}^{\rho} \tilde{p}_{\rho}+\frac{4 \Lambda^{2}}{D} e^{\frac{4}{D-2} \Phi}\right) \chi_{\theta}+\sigma_{\theta} e_{n}\left(\chi_{\rho}^{\rho}+\chi_{\theta}\right) \\
& +\frac{8(D-1)(D-4)}{D(D-2)^{2}} \Lambda^{2} e^{\frac{4}{D-2} \Phi} \chi_{\xi} \\
0 & =e_{n}^{2}\left(\chi_{\xi}\right)+\sigma e_{n}\left(\chi_{\xi}\right)-\left(\tilde{p}^{2}+\frac{D^{2}-6 D+4}{D(D-2)} \Lambda^{2} e^{\frac{4}{D-2} \Phi} \chi_{\xi}\right)+\frac{D-2}{2(D-4)} \sigma_{\theta} e_{n}\left(\chi_{\rho}^{\rho}+\chi_{\theta}\right) \\
& +\frac{D-2}{2 D} \Lambda^{2} e^{\frac{4}{D-2} \Phi} \chi_{\theta} \\
0 & =\tilde{p}^{\mu}\left(e_{n}\left(\chi_{\rho}^{\rho}+\chi_{\theta}\right)+\left(\sigma_{\rho}-\sigma_{\mu}\right) \chi_{\rho}^{\rho}+\left(\sigma_{\theta}-\sigma_{\mu}\right) \chi_{\theta}+\frac{8}{D-4} \sigma_{\theta} \chi_{\xi}\right) \\
& -\tilde{p}^{\rho}\left(e_{n}\left(\chi_{\mu \rho}\right)+\left(\sigma_{\rho}-\sigma_{\mu}\right) \chi_{\mu \rho}\right) \\
0 & =e_{n}^{2}\left(\chi_{\rho}^{\rho}+\chi_{\theta}\right)+2 \sigma_{\rho} e_{n}\left(\chi_{\rho}^{\rho}\right)+2 \sigma_{\theta} e_{n}\left(\chi_{\theta}\right)+\frac{16}{D-4} \sigma_{\theta} e_{n}\left(\chi_{\xi}\right)-\frac{8 \Lambda^{2}}{(D-2)^{2}} e^{\frac{4}{D-2} \Phi} \chi_{\xi}
\end{aligned}
$$

We note that we have left with the unknowns $\left(\chi_{\mu \nu}, \chi_{\theta \theta}, \chi_{\xi}\right)$, whose system of coupled, second order differential equations is given by the first three equations; the last two equations should work as constraints ${ }^{14}$.

\footnotetext{
${ }^{14}$ The first one of them is of first order, while that the second one can be put in first order form by using the first two equations in (B.15).
} 


\section{B.3 Holographic models of $d$ dimensional Yang-Mills theories}

Let us take, among the $x^{\mu}$ coordinates, $d$ non compact, equivalent, $x^{\alpha}$-coordinates, $\alpha=$ $0,1, \ldots, d-1$, and $D-d-2$ compact and non equivalent $\tau^{i}, i=1, \ldots D-d-2, \tau_{i} \equiv$ $\tau_{i}+2 \pi R_{i}$. We will denote with a quantities associated with the non-compact directions $\left(A_{\alpha}=\tilde{A}, a_{\alpha}=\tilde{a}, \sigma_{\alpha}=\tilde{\sigma}\right.$, etc). The metric and constraints (2.3) are,

$$
\begin{aligned}
l_{0}^{-2} G= & u^{2}\left(f(u)^{\tilde{a}} \eta_{\mu \nu} d x^{\mu} d x^{\nu}+\sum_{i} f(u)^{a_{i}} d \tau^{i 2}\right)+\frac{d u^{2}}{u^{2} f(u)}+u_{1}^{2} \frac{d \theta^{2}}{u^{2} f(u)^{a_{\theta}}} \\
& d \tilde{a}+\sum_{i} a_{i}+a_{\theta}=1 \quad, \quad d \tilde{a}^{2}+\sum_{i} a_{i}{ }^{2}+a_{\theta}{ }^{2}=1
\end{aligned}
$$

We stress that $D-d-2$ exponents remain free.

\section{B.4 Metric: transverse fluctuations}

They correspond to take the ansätz,

$$
\chi_{\alpha \beta}(u)=\epsilon_{\alpha \beta}(p) \chi(u) \quad ; \quad \epsilon_{\alpha}^{\alpha}=0, \epsilon_{\alpha \beta} p^{\beta}=0
$$

and the rest of the fluctuations zero. This ansätz solves (B.15) if $\chi$ satisfies,

$$
e_{n}^{2}(\chi)+\sigma e_{n}(\chi)+\left(\frac{M^{2}}{\tilde{A}^{2}}-\sum_{i} \tilde{p}_{i}^{2}\right) \chi=0
$$

where $M^{2} \equiv-p^{\alpha} p_{\alpha}$ is the $d$-dimensional mass. By making the change (4.19), it turns out that $\mathrm{H}$ satisfy exactly the equation (5.8).

\section{B.5 Metric: longitudinal fluctuations}

They correspond to take the ansätz, at fixed $i$ (but for any $i$ ),

$$
\chi_{i \alpha}(u)=\epsilon_{\alpha}(p) \chi(u) \quad ; \quad p_{i}=0, \epsilon_{\alpha} p^{\alpha}=0
$$

and the rest of the fluctuations zero. It is consistent with (B.15) if $\chi(u)$ obeys,

$$
e_{n}^{2}(\chi)+\sigma e_{n}(\chi)+\left(\frac{M^{2}}{\tilde{A}^{2}}-\sum_{i} \tilde{p}_{i}^{2}-\left(\tilde{\sigma}-\sigma_{i}\right)^{2}\right) \chi=0
$$

By making the change (4.19), it turns out that $\mathrm{H}$ satisfy exactly the equation (5.10).

\section{B.6 Scalar fluctuations}

We expect that they come from the dilaton and the metric. However, at difference of the case worked out in references [34], [18], they do not disentangle, in the sense that putting to zero the metric fluctuations is not consistent. So we start with the ansätz for the metric,

$$
\chi_{\mu \nu}(u)=a_{\mu}(u) \eta_{\mu \nu}+b_{\mu \nu}(u) p_{\mu} p_{\nu}
$$


which at difference of the cases treated above, is not transverse neither traceless. It results convenient to introduce the following variables,

$$
\begin{aligned}
\chi_{\mu} & \equiv a_{\mu}-\sigma_{\mu} \frac{\chi_{\theta}}{\sigma_{\theta}} \\
\tilde{\chi}_{\mu \nu} & \equiv A_{\mu} A_{\nu} b_{\mu \nu}-\frac{1}{2}\left(A_{\mu}^{2} b_{\mu \mu}+A_{\nu}^{2} b_{\nu \nu}\right) \\
\tilde{\chi}_{\mu} & \equiv A_{\mu}^{2} e_{n}\left(b_{\mu \mu}\right)-\frac{\chi_{\theta}}{\sigma_{\theta}} \\
\tilde{\chi}_{\theta} & \equiv e_{n}\left(\frac{\chi_{\theta}}{\sigma_{\theta}}\right) \\
\tilde{\chi}_{\xi} & \equiv \chi_{\xi}-\frac{D-2}{2(D-4)} \chi_{\theta}
\end{aligned}
$$

We note that $b_{\mu \nu}$ is replaced by $\tilde{\chi}_{\mu \nu}$ and $\tilde{\chi}_{\mu}$ (by construction, $\tilde{\chi}_{\mu \mu} \equiv 0$ for any $\mu$ ). With them, and combining equations in (B.15), we can rearrange the system in the following way,

$$
\begin{aligned}
0 & =e_{n}^{2}\left(\chi_{\mu}\right)+\sigma e_{n}\left(\chi_{\mu}\right)-\tilde{p}^{\rho} \tilde{p}_{\rho} \chi_{\mu}+\frac{2 \Lambda^{2}}{D-2} e^{\frac{4}{D-2} \Phi}\left(1+\frac{D-4}{D} \frac{\sigma_{\mu}}{\sigma_{\theta}}\right) \tilde{\chi}_{\theta} \\
& -\frac{8 \Lambda^{2}}{(D-2)^{2}} e^{\frac{4}{D-2} \Phi}\left(1+(D-1) \frac{D-4}{D} \frac{\sigma_{\mu}}{\sigma_{\theta}}\right) \tilde{\chi}_{\xi} \\
0 & =e_{n}^{2}\left(\tilde{\chi}_{\mu \nu}\right)+\left(\sigma-2\left(\sigma_{\mu}+\sigma_{\nu}\right)\right) e_{n}\left(\tilde{\chi}_{\mu \nu}\right)+\sum_{\rho} \tilde{p}^{\rho} \tilde{p}_{\rho}\left(\tilde{\chi}_{\mu \rho}+\tilde{\chi}_{\nu \rho}-\tilde{\chi}_{\mu \nu}\right) \\
& +\left(4 \sigma_{\mu} \sigma_{\nu}-\frac{2 \Lambda^{2}}{D-2} e^{\frac{4}{D-2} \Phi}\right) \tilde{\chi}_{\mu \nu}+\tilde{\chi}_{\theta}+\chi_{\mu}+\chi_{\nu}-\sum_{\rho} \chi_{\rho} \\
& +\frac{1}{2}\left(e_{n}\left(\tilde{\chi}_{\mu}+\tilde{\chi}_{\nu}\right)+\sigma\left(\tilde{\chi}_{\mu}+\tilde{\chi}_{\nu}\right)\right)-\sigma_{\mu} \tilde{\chi}_{\nu}-\sigma_{\nu} \tilde{\chi}_{\mu} \\
0 & =e_{n}^{2}\left(\tilde{\chi}_{\xi}\right)+\sigma e_{n}\left(\tilde{\chi}_{\xi}\right)-\left(\tilde{p}^{2}+\Lambda^{2} e^{\frac{4}{D-2} \Phi}\right) \tilde{\chi}_{\xi} \\
0 & =\sigma_{\theta} e_{n}\left(\tilde{\chi}_{\theta}\right)+2 D^{2}\left(\ln A_{\theta}\right) \tilde{\chi}_{\theta}+\sigma_{\theta} e_{n}\left(\sum_{\rho} \chi_{\rho}\right)+\sigma_{\theta} \sum_{\rho} \tilde{p}^{\rho} \tilde{p}_{\rho} \tilde{\chi}_{\rho} \\
& +\frac{8(D-1)(D-4)}{D(D-2)^{2}} \Lambda^{2} e^{\frac{4}{D-2} \Phi} \tilde{\chi}_{\xi} \\
0 & =\sum_{\rho}\left(e_{n}\left(\chi_{\rho}\right)+\left(\sigma_{\rho}-\sigma_{\mu}\right) \chi_{\rho}\right)-e_{n}\left(\chi_{\mu}\right)+\left(\sigma-\sigma_{\mu}\right) \tilde{\chi}_{\theta}+\frac{8}{D-4} \sigma_{\theta} \tilde{\chi}_{\xi} \\
& -\sum_{\rho} \tilde{p}^{\rho} \tilde{p}_{\rho}\left(\frac{1}{2}\left(\tilde{\chi}_{\mu}-\tilde{\chi}_{\rho}\right)+e_{n}\left(\tilde{\chi}_{\mu \rho}\right)-2 \sigma_{\mu} \tilde{\chi}_{\mu \rho}\right) \\
0 & =\sum_{\rho} \tilde{p}^{\rho} \tilde{p}_{\rho}\left(\chi_{\rho}+\left(\sigma-\sigma_{\rho}\right) \tilde{\chi}_{\rho}+\sum_{\sigma} \tilde{p}^{\sigma} \tilde{p}_{\sigma} \tilde{\chi}_{\rho \sigma}\right)+\frac{D-2}{D} \Lambda^{2} e^{\frac{4}{D-2} \Phi}\left(\tilde{\chi}_{\theta}-\frac{8}{(D-2)^{2}} \tilde{\chi}_{\xi}\right)
\end{aligned}
$$

We observe that the equation for $\tilde{\chi}_{\xi}$ gets decoupled from the rest of the fluctuations, so we can divide the scalar fluctuations in two disjoint cases.

$\tilde{\chi}_{\xi} \neq 0$

If so, the equation that defines the spectra is just,

$$
0=e_{n}^{2}\left(\tilde{\chi}_{\xi}\right)+\sigma e_{n}\left(\tilde{\chi}_{\xi}\right)-\left(\tilde{p}^{2}+\Lambda^{2} e^{\frac{4}{D-2} \Phi}\right) \tilde{\chi}_{\xi}
$$


By making the change (4.19), it turns out that $\mathrm{H}$ satisfy exactly the equation (4.20)-(4.21) corresponding to the dilatonic fluctuations. We stress here that, obtained the solution to (B.24) for $\tilde{\chi}_{\xi}$, one should put it in the remaining equations in (B.23) and solve for the rest of the perturbations that define this fluctuation, because putting them to zero is not consistent.

$\tilde{\chi}_{\xi}=0$

The equation $(\overline{\mathrm{B} .24})$ is trivially satisfied, and we remain with a couple set of equations obtained by putting $\tilde{\chi}_{\xi}=0$ in $(\overline{B .23})$. This system should be equivalent to (5.15)-(5.16) that defines the scalar fluctuation of the metric; the analysis of this equivalence becomes cumbersome and we have not verified it.

\section{References}

[1] J. M. Maldacena, Adv. Theor. Math. Phys. 2, 231 (1998) [Int. J. Theor. Phys. 38, 1113 (1999)] arXiv:hep-th/9711200.

[2] S. S. Gubser, I. R. Klebanov and A. M. Polyakov, Phys. Lett. B 428, 105 (1998) arXiv:hep-th/9802109.

[3] E. Witten, Adv. Theor. Math. Phys. 2, 253 (1998) arXiv:hep-th/9802150.

[4] O. Aharony, S. S. Gubser, J. M. Maldacena, H. Ooguri and Y. Oz, Phys. Rept. 323, 183 (2000) arXiv:hep-th/9905111, and references therein.

[5] E. Imeroni, arXiv:hep-th/0312070. and references therein.

[6] E. Witten, "Anti-de Sitter space, thermal phase transition, and confinement in gauge theories," Adv. Theor. Math. Phys. 2, 505 (1998) arXiv:hep-th/9803131.

[7] D. J. Gross and H. Ooguri, Phys. Rev. D 58, 106002 (1998) arXiv:hep-th/9805129.

[8] C. Csaki, H. Ooguri, Y. Oz and J. Terning, JHEP 9901, 017 (1999) arXiv:hep-th/9806021.

[9] R. de Mello Koch, A. Jevicki, M. Mihailescu and J. P. Nunes, Phys. Rev. D 58, 105009 (1998) arXiv:hep-th/9806125.

[10] A. Hashimoto and Y. Oz, Nucl. Phys. B 548, 167 (1999) arXiv:hep-th/9809106.

[11] J. A. Minahan, JHEP 9901, 020 (1999) arXiv:hep-th/9811156.

[12] R. C. Brower, S. D. Mathur and C. I. Tan, Nucl. Phys. B 574, 219 (2000) arXiv:hep-th/9908196.

[13] R. C. Brower, S. D. Mathur and C. I. Tan, Nucl. Phys. B 587, 249 (2000) arXiv:hep-th/0003115.

[14] E. Caceres and R. Hernandez, Phys. Lett. B 504, 64 (2001) arXiv:hep-th/0011204.

[15] A. M. Polyakov, Nucl. Phys. Proc. Suppl. 68, 1 (1998) arXiv:hep-th/9711002.

[16] D. Kutasov and N. Seiberg, Phys. Lett. B 251, 67 (1990).

[17] D. Kutasov, arXiv:hep-th/9110041.

[18] S. Kuperstein and J. Sonnenschein, JHEP 0407, 049 (2004) arXiv:hep-th/0403254.

[19] S. Kuperstein and J. Sonnenschein, JHEP 0411, 026 (2004) arXiv:hep-th/0411009. 
[20] A. M. Polyakov, Int. J. Mod. Phys. A 14, 645 (1999) arXiv:hep-th/9809057.

[21] A. R. Lugo and M. B. Sturla, Nucl. Phys. B 792, 136 (2008) arXiv:0709.0471 [hep-th]].

[22] J. Sonnenschein, arXiv:hep-th/0003032.

[23] S. K. Ashok, S. Murthy and J. Troost, Nucl. Phys. B 749, 172 (2006) arXiv:hep-th/0504079.

[24] A. Fotopoulos, V. Niarchos, N. Prezas, JHEP 0510, 081 (2005). arxiv:hep-th/0504010

[25] See for example, C. V. Johnson, arXiv:hep-th/0007170, and references therein.

[26] L. Susskind and E. Witten, arXiv:hep-th/9805114.

[27] J. M. Maldacena, Phys. Rev. Lett. 80, 4859 (1998) arXiv:hep-th/9803002.

[28] S. J. Rey and J. T. Yee, Eur. Phys. J. C 22, 379 (2001) arXiv:hep-th/9803001.

[29] Y. Kinar, E. Schreiber and J. Sonnenschein, Nucl. Phys. B 566, 103 (2000) arXiv:hep-th/9811192.

[30] S. Murthy, JHEP 0311, 056 (2003) arXiv:hep-th/0305197.

[31] S. M. Carroll, "Lecture notes on General Relativity," arXiv:gr-qc/9712019.

[32] J. M. Bardeen, Phys. Rev. D 22, 1882 (1980).

[33] K. Nakamura, Prog. Theor. Phys. 117, 17 (2007) arXiv:gr-qc/0605108.

[34] N. R. Constable and R. C. Myers, JHEP 9910, 037 (1999) arXiv:hep-th/9908175.

[35] A. R. Lugo and M. B. Sturla, Phys. Lett. B 637, 338 (2006) arXiv:hep-th/0604202.

[36] J. Polchinski, "String theory, vol. 1 and 2", Cambridge University Press, Cambridge (1998).

[37] N. Itzhaki, J. M. Maldacena, J. Sonnenschein and S. Yankielowicz, Phys. Rev. D 58, 046004 (1998) arXiv:hep-th/9802042.

[38] J. M. Maldacena and C. Nunez, Phys. Rev. Lett. 86, 588 (2001) arXiv:hep-th/0008001.

[39] I. R. Klebanov and A. A. Tseytlin, Nucl. Phys. B 578, 123 (2000) arXiv:hep-th/0002159.

[40] I. R. Klebanov and M. J. Strassler, JHEP 0008, 052 (2000) arXiv:hep-th/0007191.

[41] M. J. Teper, Phys. Rev. D 59, 014512 (1999) arXiv:hep-lat/9804008.

[42] C. J. Morningstar and M. J. Peardon, Phys. Rev. D 60 (1999) 034509 arXiv:hep-lat/9901004.

[43] U. Gursoy, E. Kiritsis and F. Nitti, JHEP 0802, 019 (2008) arXiv:0707.1349 [hep-th]].

[44] E. Kiritsis, arXiv:0901.1772 [hep-th], and references therein. 